\title{
الريف البرازيلي أنموذجا للتنمية في سياق الاقتصاد الأخضر بعد عام 2003(1) تأليف: لورو ماتي
}

$$
\text { ترجمة وتعليق: ا.م. د قاسم شعيب السلطاني }
$$

Qassimalsultani@yahoo.com

07700169262

\section{ABSTRACT:}

This paper discusses the Brazilian rural development model in the context of Green Economy and the sustainable development debate, issues that have be discussed in international sphere. this paper will try to answer the following question: Is the green economy approach a good way to promote sustainable development in countries like Brazil? To answer this question we are concentrating our analysis in the agricultural sector. we analyzed the Brazilian rural development model and the public investment in the agricultural and livestock sector, seeking to emphasize the government option towards primary commodity production for international markets. The main conclusions of the paper is that to understand the Brazilian society today we need to take account a transition from an agrarian society to an industrial society, a process that occurred in Brazil during the 20th Century. This path Brazilian society has mapped since its inception is marked by the contradiction between abundance for a few portions of the population and famine for the great majority. if the year 2011 showed Brazil to be the 6th largest economy in the world, at the same time the world and the greatest rates of social exclusion.

keyword: Brazil, sustainable development, Brazilian rural, Brazil Economy, Green Economy.

الملخص

يناقش البحث نموذج التنمية الريفية في البرازيل في سياق الاقتصاد الأخضر والجدال بخصوص التنمية المستدامة والقضايا التي نوقشت في الجمال الدولي.

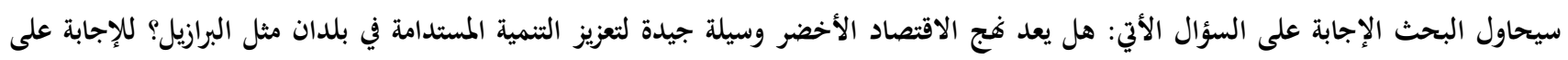

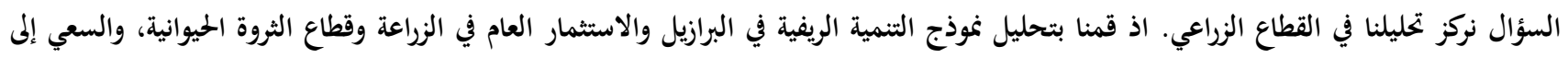

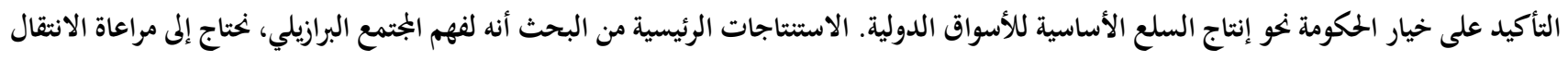

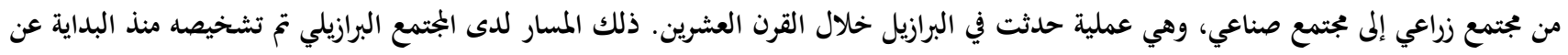
طريق التناقض بين الوفرة بالنسبة لعدد قليل من السكان والمجاعة بالنسبة للغالبية العظمى، إذا أظهر عام وفي ذات الوقت الذي شهدت فيه أكبر معدلات الاستبعاد الاجتماعي. الكلمات المفتاحية: البرازيل، تنمية مستدامة، الاقتصاد الأخضر، الريف البرازيلي، القطاع الزراعي.

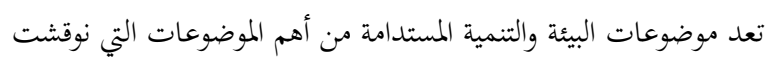
المقدمة:

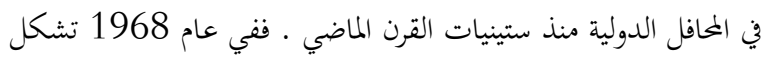


عندما كتبت وثيقة جديدة عرفت ب "بروتوكول كيوتو" . تم اقتراح بجموعة

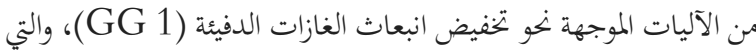
تسلط الضوء على القواعد الأساسية في إنشاء وتطوير سوق الائتمان

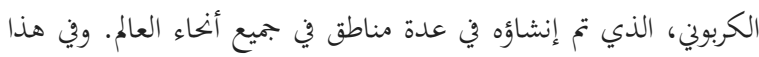

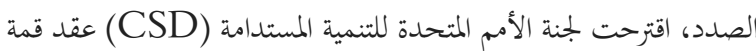

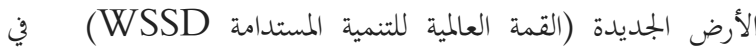

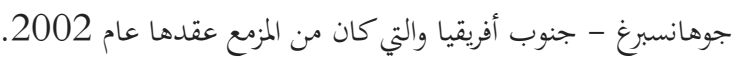
كان المدف الرئيسي من المؤتمر استعراض الأهداف المقترحة لأجندة أعمال

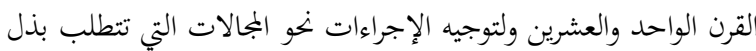

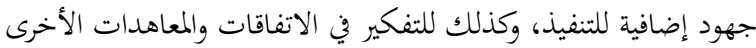

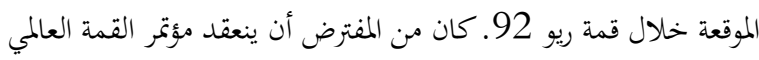

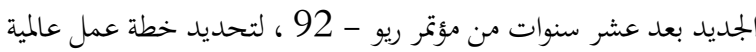
قادرة على التوفيق بين الاحتياجات المشروعة للبشرية التنمية الاقتصادية

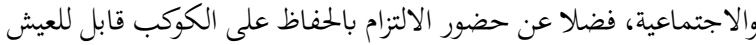

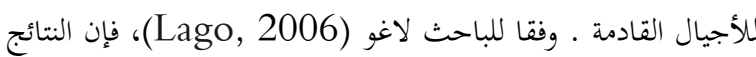

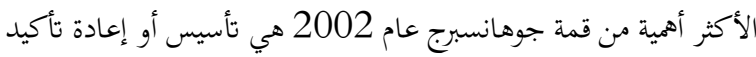
أهداف القضاء على الفقر، شبكات المياه وأنظمة الصرف الصحي،

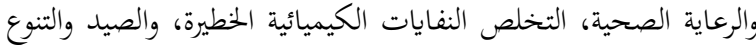
البيولوجي، مع تضمين موضوعين حملا تحديات كبرى عن السابق، كانت: مفاوضات (الطاقات المتجددة ومحاسبة الشركات) سياسيا، قرار إنشاء صندوق تضامن عالمي للقضاء على الفقر والتآزر بين مختلف الجهات الفاعلة الاجتماعية نحو جعل مشاريع الشراكة أكثر ديناميكية وفعالية. كان من المقرر عقد مؤتمر ريو +20 بعد عشر سنوات من مؤتمر القمة

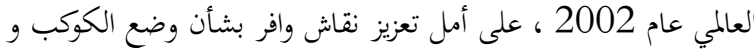
المسارات الحالية للحضارة، بدءا من منظور التعبير عن أهداف الجهات

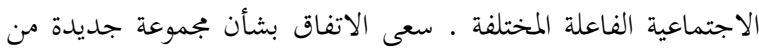

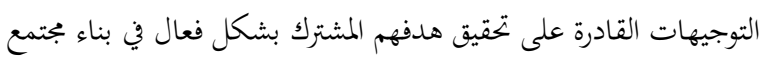

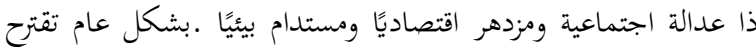

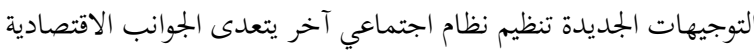

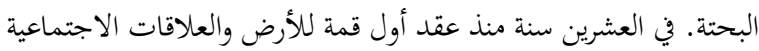

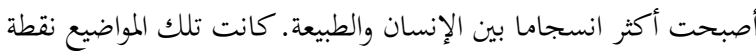
الانطلاق لمؤتمر ريو -20. يجب إعادة تأكيد الغالبية العظمى من المعايير

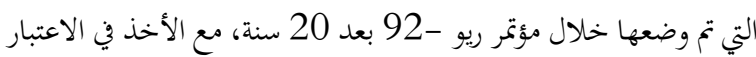

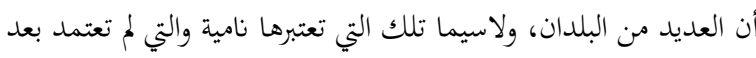

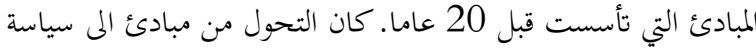

يجذر من عدم استمرار التنمية الإقتصادية بسبب نفاد الموارد كنتيجة للزيادة السكانية المتسارعة و قد أكدت

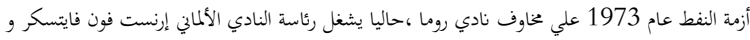

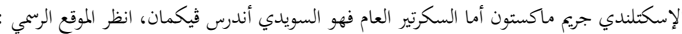
http://www.clubofrome.org/
نادي روما(*)، بهدف مناقشة وتحليل حدود زياد النمو الاقتصادي القائم على الموارد

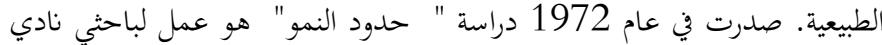
روما بقيادة دنيس مداوس Dennis Meadaows. في ذلك البحث، ناقشوا فكرة انه بسبب محدودية الموارد الطبيعة للكوكب والاستقرار الاقتصادي، لاسيما ندرة

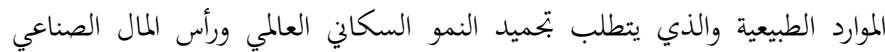

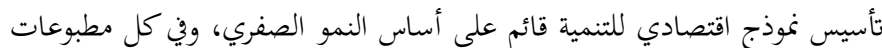
نادي روما ان مثل ذلك السيناريو سيكون السبيل الوحيد لتجنب كارثة بيئية وشيكة.

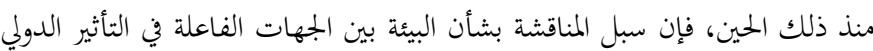
تضاعفت على هذا النحو ونشأت مفاهيم متنوعة من أجل استيعاب عدد وافر من الموضوعات والخصوصيات البيئية، وكان مفهوم التنمية المستدامة من أكثرها تكرارا.

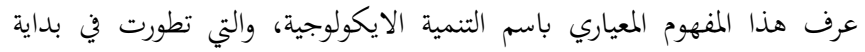

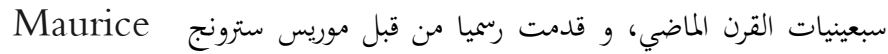

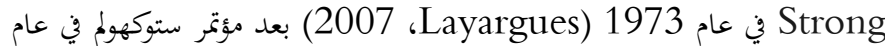

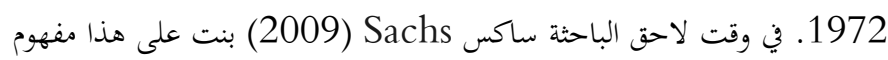
من خلال إنشاء جدول لاستراتيجيات الاستدامة على ثلاث ركائز أساسية: الكفاءة

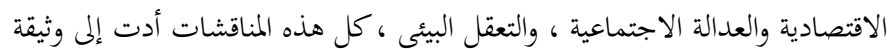
تسمى "تقرير برونتلاند" Brundtland Report ، من إعداد اللجنة العالمية المعنية بالبيئة والتنمية ونشرت في عام 1987. تمت كتابة التقرير وسط بمموعة من التران

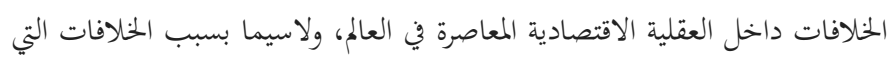

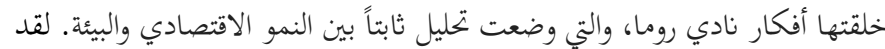

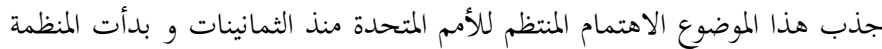

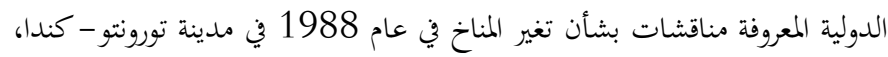

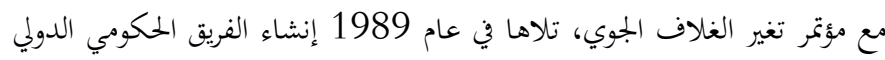

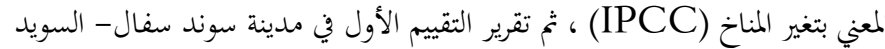

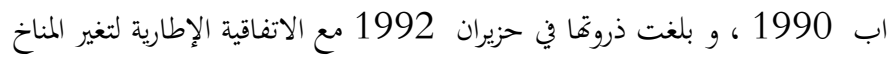
ECO-92 (UNFCCC)

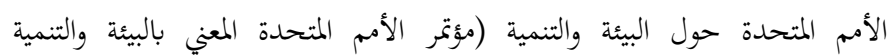

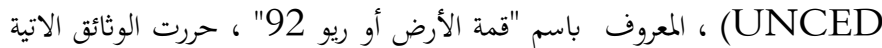

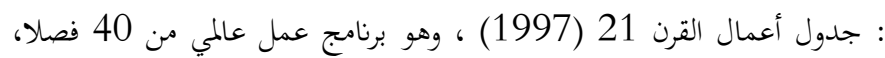

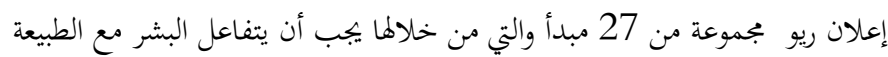
، مبادئ الغابات اتفاقية التنوع البيولوجي والاتفاقية الإطارية لتغير المناخ. تلك الوثائقي،

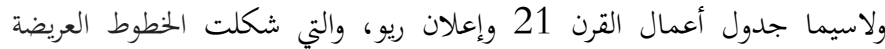

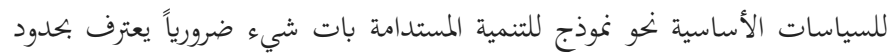

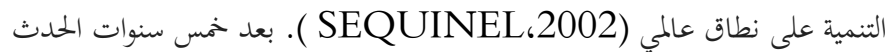
الذي أضفى الشرعية على مشاكل الاحتباس الحراري كان في مدينة كيوتو - اليابان

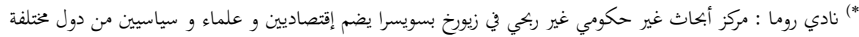

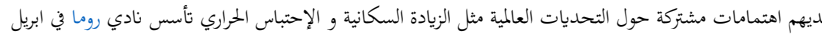

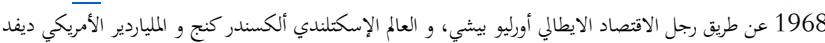

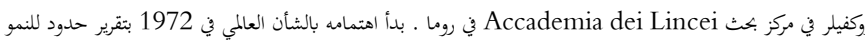
LTG الذي بيعت منه 30 مليون نسخة و قد ترجم ل 30 لغة مما جعله أكثر كتب الاقتصاد البيئي مبيعا في التاريخ و 
للوزراء، فأن الاقتصاد الأخضر والنمو عملية متكاملة، و تتطلب من منظمة التعاون الاقتصادي والتنمية (OECD) إنشاء استراتيجية للنمو

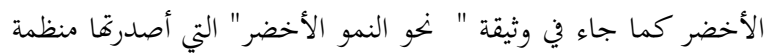
التعاون الاقتصادي والتنمية في أيار 2011 ـ نشرت إدارة الأمم المتحدة للشؤون الاقتصادية والاجتماعية (UNDESA) في كانون الأول من عام 2009 مذكرة فنية بعنوان "فج عالمي اخضر جديد للمناخ والطاقة والتنمية " . فيما نشر مجلس الأعمال العالمي للتنمية المستدامة في عام

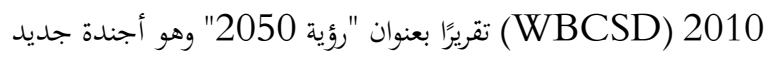
للأعمال، وقعت عليه 29 شركة كبيرة تشكل جزءًا من المنظمة. تم اقتراح

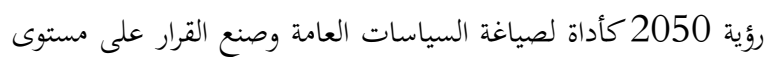
الأربعين عام القادمة. وفي عام 2011 أصدر برنامج الأمم المتحدة للبيئة تقريرا جديدا بعنوان " نحو الاقتصاد الأخضر: الطرق المؤدية إلى التنمية

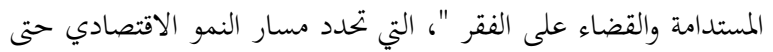
عام 2050. إنها الوثيقة الأساسية التي تنوي هذه المنظمة مناقشتها خلال مؤتمر ريو +20. هذا التقرير هو من بين المساهمات الرئيسية لبرنامج الأمم

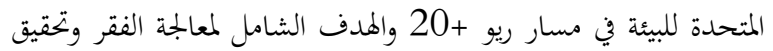

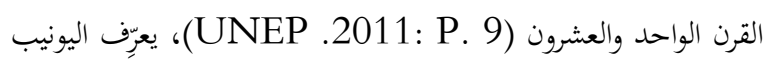
الاقتصاد الأخضر بأنه : " اقتصاد يؤدي إلى تحسين الرفاهية البشرية

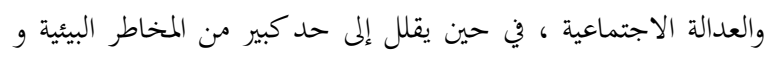
النقص الإيكولوجي (UNEP"2011: P. 5-6) . . ومع ذلك ، بعض الخطوط ألأخرى على طول ذلك ، وهذا تعريف يوفر بعض القياسات ضمن نفس النموذج الجاري من الهيمنة الطبيعية والاستكشاف:

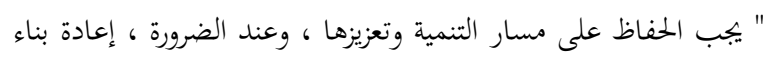

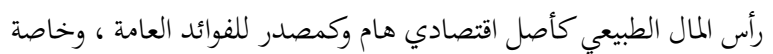

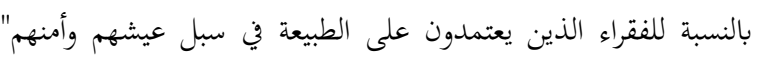

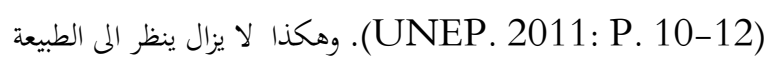
كأحد الأصول الاقتصادية. طوال نشر هذه الوثائق ، يمكن أن يرى أن برنامج الأمم المتحدة للبيئة

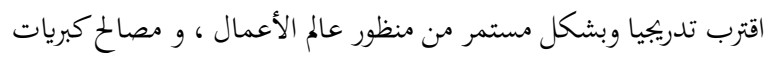
الشركات ، سواء من خلال الشراكات وتنظيم مختلف الأحداث. يبدو واضحاً أن القاسم المشترك بين كل تلك الوثائق البحث عن إستراتيجية

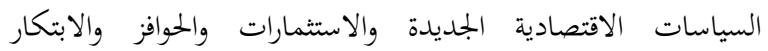
التكنولوجي بالتوافق مع السياسات البيئة، لذلك يمكن أن يتعافى الاقتصاد وخلق فرص عمل جديدة، أكثر من أي وقت مضى، وسعي إلى نمو رأس بلى بلى

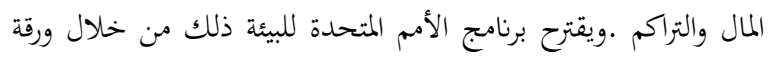

عامه واحدا من أصعب العمليات التي تم التفاوض عليها والوصول الى اتفاق بين رؤساء الدول والحكومات الحاضرين في المؤتمر الأخير في عام 2012 هذا من ناحية، من ناحية أخرى، كانت جميع تلك الأحداث حاسمة لإدخال منظور التنمية المستدامة في صميم قرارات الأمم المتحدة بحيث تكون متكاملة بسلاسة مع استراتيجيات التنمية الوطنية والدولية. في هذه الحالة، السؤال الكبير الذي طرحه برنامج الأمم المتحدة للبيئة في السنوات الأخيرة: كيف يمكننا أن نلبي الزيادة في عدد سكان العالم دون استنزاف الموارد الطبيعية؟ لمبيج مع وضع تلك السيناريوهات في الاعتبار، سيحاول هذا البحث الإجابة عن السؤال الاتي :هل يقترب الاقتصاد الأخضر من طريقة جيدة لتعزيز التنمية المستدامة في دولة مثل البرازيل؟ لإججابة على هذا، ركزت البحث تحليله في القطاع الزراعي. من أجل الوصول إلى هذه الأهداف ، تم ترتيب هذا الفصل على النحو التالي: أولا

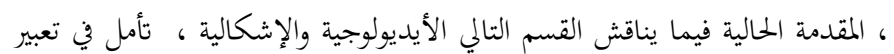
"الاقتصاد الأخضر" ، مع مناقشة الآراء في سياق تاريخي ، يقدم القسم الثاني مناقشة موجزة عن نموذج التنمية في البرازيل ، ويحاول تسليط الضوء على التناقضات والتحديات ضمن منظور التنمية المستدامة ، يحلل القسم الثالث نموذج التنمية الريفية

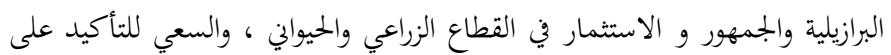

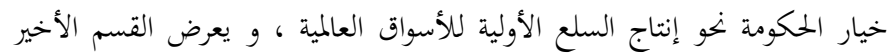

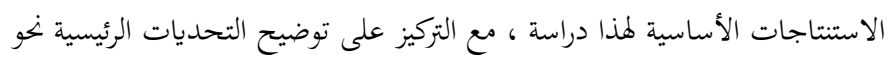
بناء نموذج للتنمية المستدامة. 1. المنهج العام للاقتصاد الأخضر: المفاهيم والنقد فيما يتعلق "بالاقتصاد الأخضر " المنهر

عرّف برنامج الأمم المتحدة للبيئة الاقتصاد الأخضر بأنه: " الاقتصاد الذي من

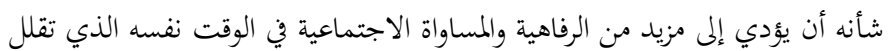
فيه بدرجة كبيرة المخاطر والندرة البيئية ". ويتم تحقيق ذلك من خلال دعمها لثاهيه لثلاثة ركائز أساسية: اقتصاد منخفض الكربون، والاستخدام الفعال للموارد الطبيعية، والاندماج الاجتماعي .وفقاً لبيريت Peret (2012) ، سعت سلسلة من وثائق الأمم المتحدة إلى بلورة أساس مفاهيمي للاقتصاد الأخضر،إذ أصدر برنامج الأمم المتحدة للبيئة في شباط 2009 ، مجموعة سياسات مقترحه تسعى لمكافحة الاحترار

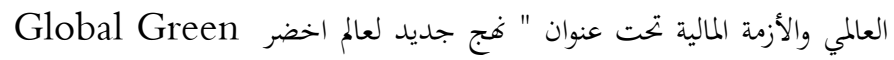
New Deal أطلقه فرانكلين دي روزفلت(*)، رئيس الولايات المتحدة الأمريكية خلال الكساد الاقتصادي في عام 1929. وقع وزراء من 34 دولة في حزيران 2009، على إعلان " النمو الأخضر"، وشهدوا بذلك على أن: " الانتعاش والنمو الاقتصادي المستدام بيئيا واجتماعيا هي التحديات

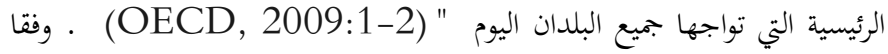

(*) فرانكلين ديلانو روزفلت: 1882- 1945 ، ، رجل دولة وزعيم سياسي أمريكي شغل منصب الرئيس الثاني والثالاثين كلولايات المتحدة من عام 1933 حتى وفاته في عام 1945 1945. روزفلت هو سياسي ديمقراطي، وفاز في في أربعة انتخابات رئاسية

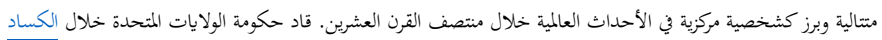

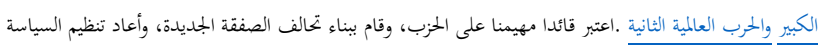

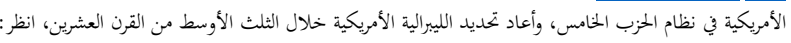


يكون التركيز إيجابيا إذا كان يقدم حدودا قادرة على أن تؤدي بشكل فعال

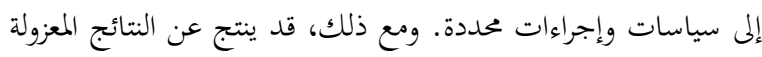

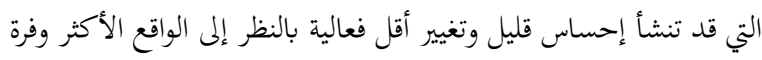

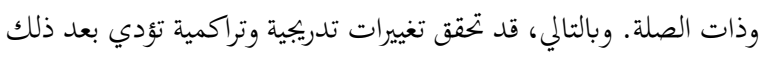

إلى تغييرات هيكلية عميقة. ولكن قد يلهم أيضًا تغييرات غير جوهرية.

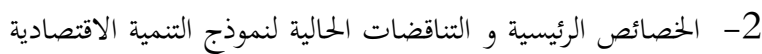

في البرازيل

بعد عقود عدة من الركود، تبنى الاقتصاد البرازيلي تغييرات مهمة اعتبارا

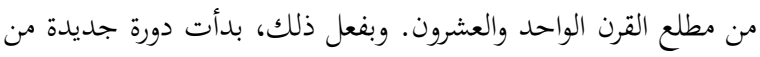
النمو، والتي أدارت بعض الإجراءات الحكومية نهو مكافحة عدم المساواة

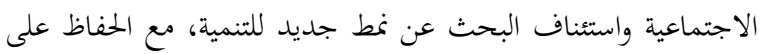
الظروف الهيكلية الميطة للنظام الاقتصادي. تم تعزيز تلك المرحلة الجديدة

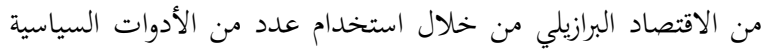

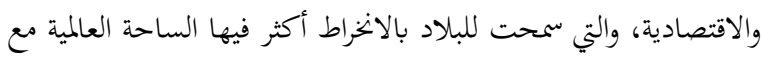

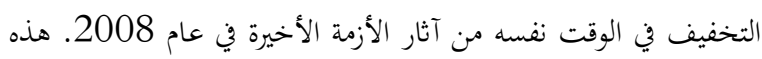

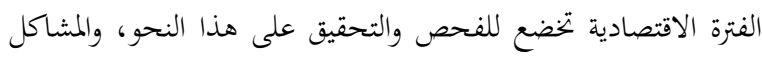

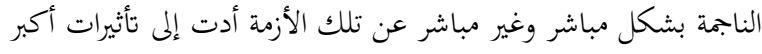

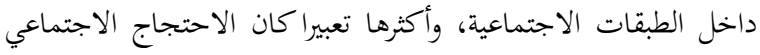

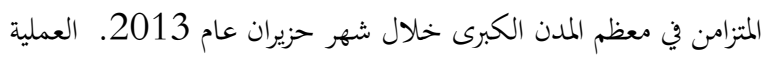
الحالية التي تنطوي على احتجاجات اجتماعية، عززت الأسطورة القائلة

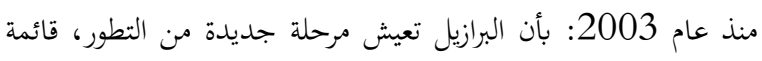

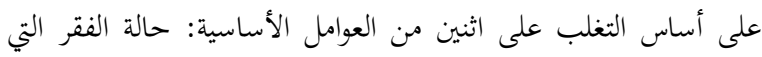
يعيشها جزء كبير من السكان والاعتماد المتطرف على رأس المال الأجنبي. هذه الموجة من التفاؤل، واتتي كانت سائدة حتى وقت قريب، غطت في الحقيقة على بجموعة التناقضات والهشاشة داخل النموذج البرازيلي، والتي

$$
\text { كانت منتشرة لسنوات. }
$$

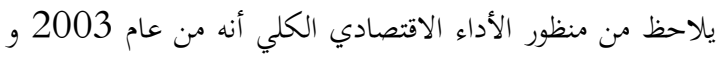

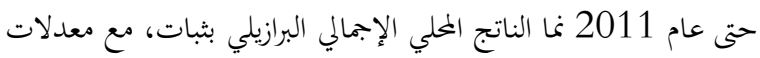

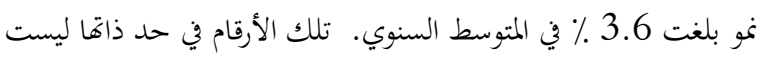

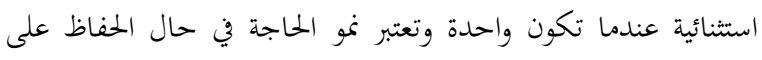

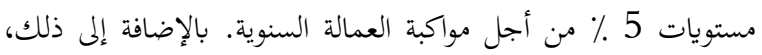

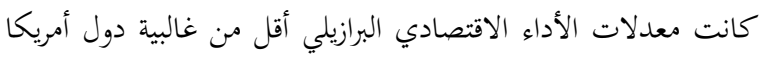

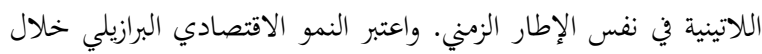

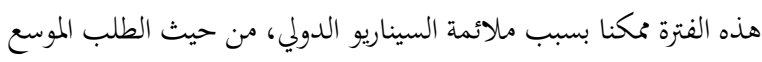

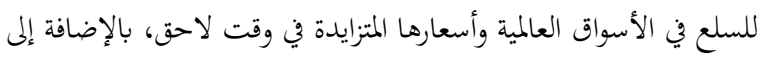

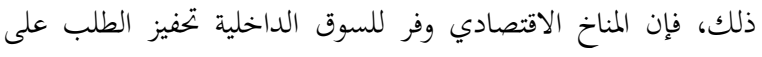

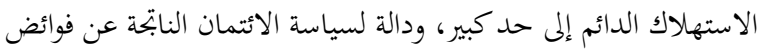
السيولة الدولية خلال عام 2008.
المناقشة الخاصة به: " فج جديد لعالم اخضر "، ان الانتعاش الاقتصادي العلمي

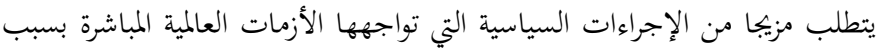
تغير المناخ والغموض في مستقبل الوقود و تزايد ندرة المياه النظيفة، وتدهور النظم

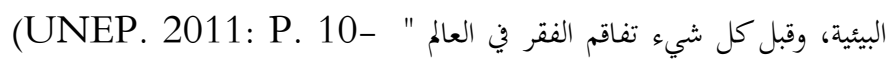

الغرض هو تقليل الاعتماد على الوقود الكربوني، وماية النظم البيئية ومصادر

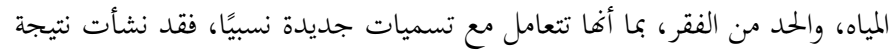

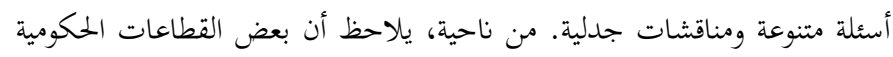

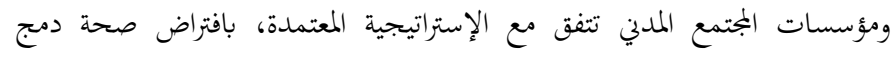

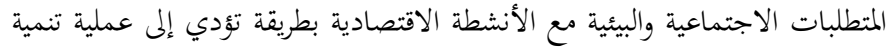

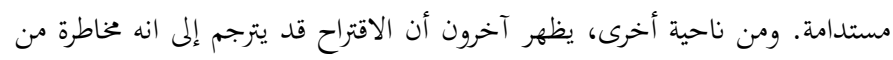

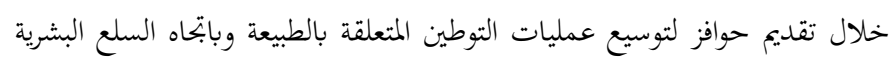

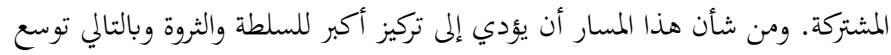

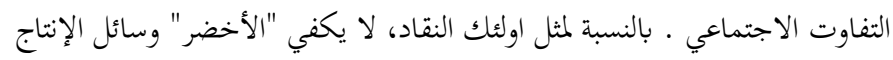

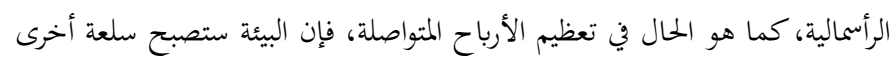

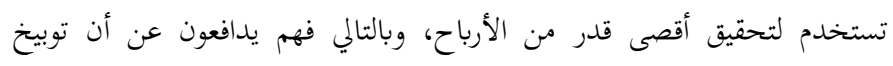

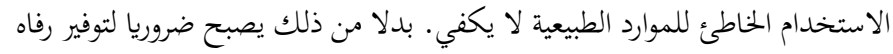

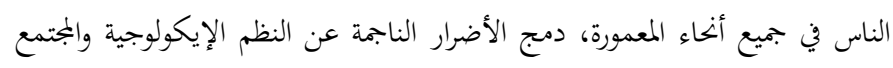

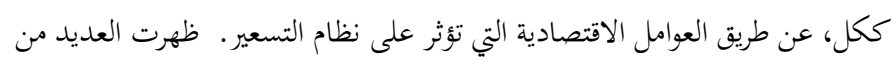

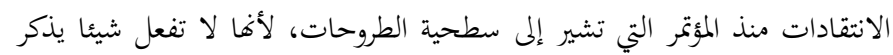

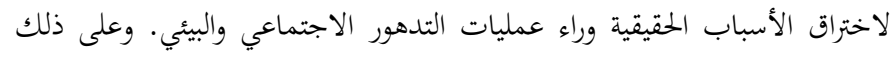

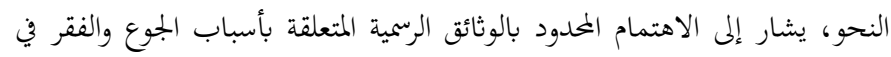

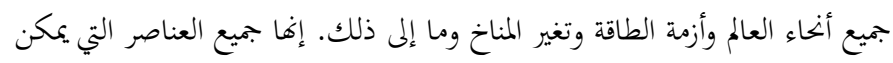

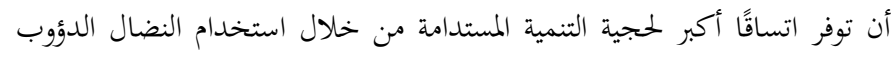

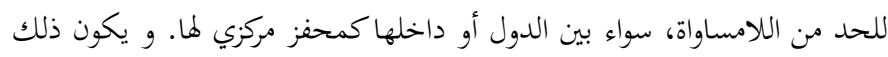

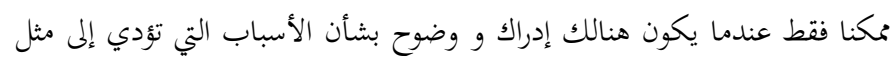

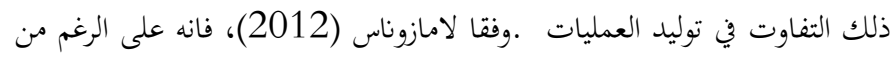

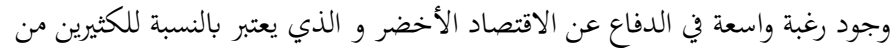

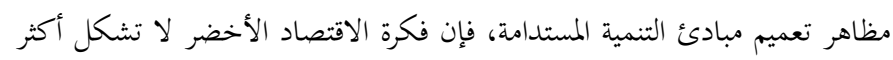

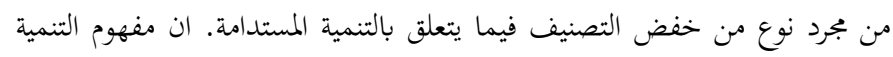

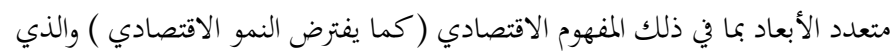

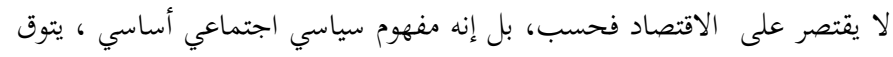

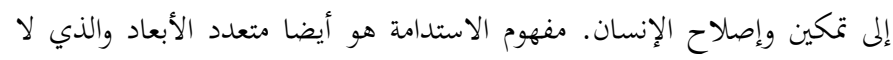

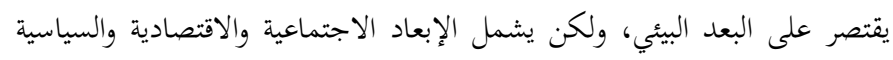

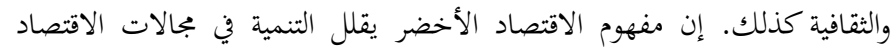

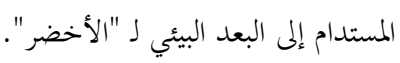
بالنسبة للمؤلف فإن تعريف الاقتصاد الأخضر هو خطوة إلى الوراء من التنمية

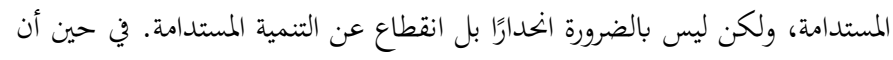

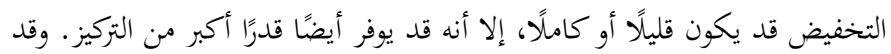


حتى مع التركيز على أهمية عملية التوسع في الإنفاق الحكومي في المجال

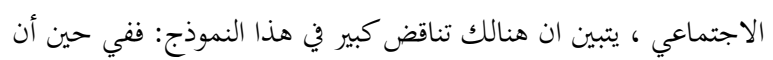

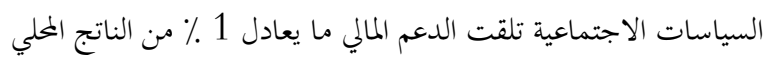

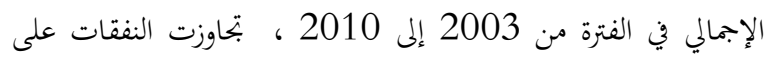

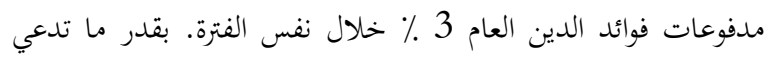

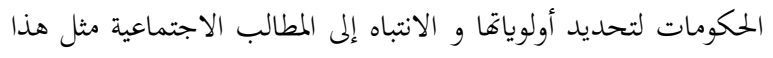

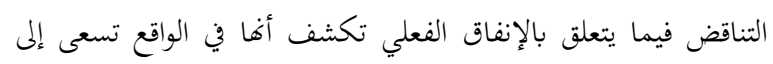

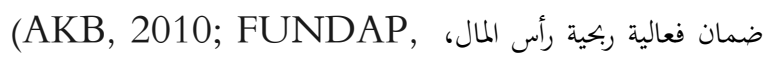
2011; IPEA, 2012).

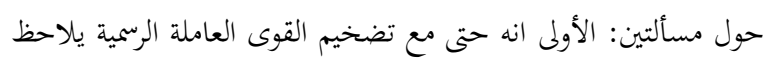

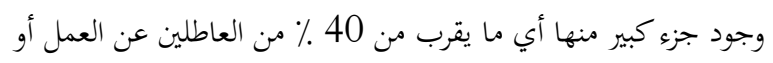

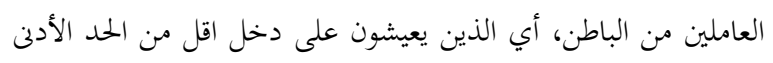

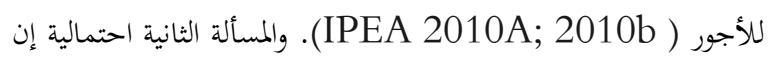
أكثر من 30 مليون شخص يعيشون تحت خط الفقر ، وهو رقم أكبر

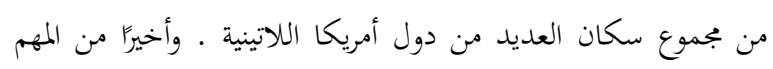

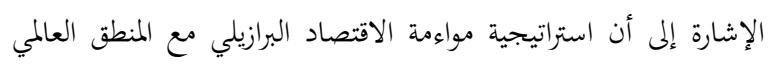

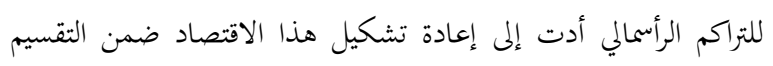

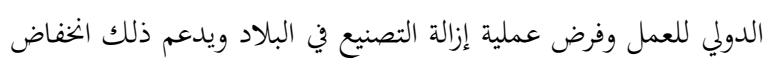

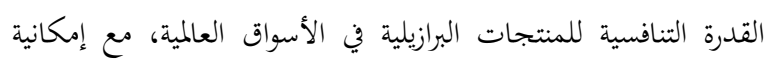

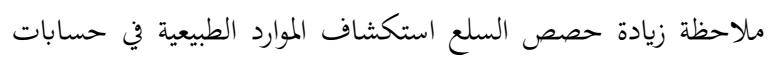

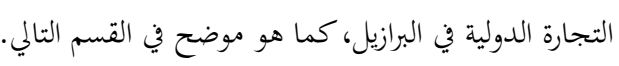
2.2 - إستراتيجية تصدير السلع الأولية

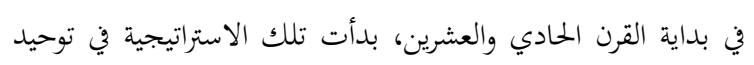

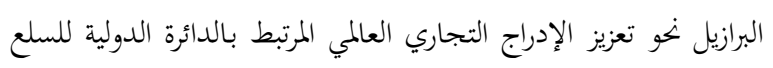

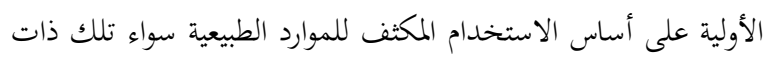
الطبيعة الزراعية او تلك المستمدة من أنشطة تعدين المعادن الاستخراجية.

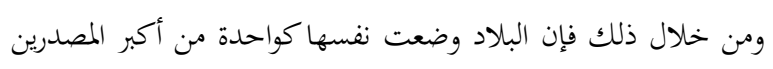

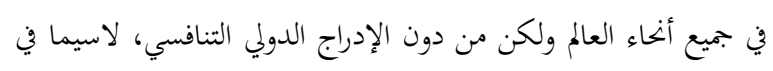

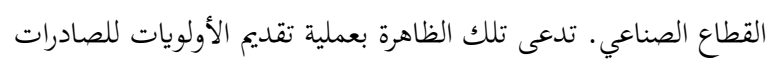

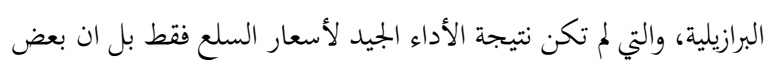

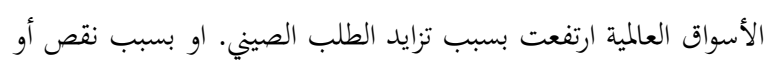

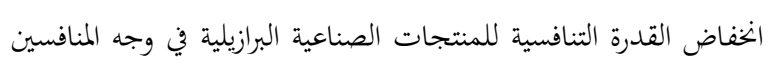
داخل التجارة الدولية. إن الانخفاض في المنتجات ذات القيمة الإجمالية الأكبر في أجندة

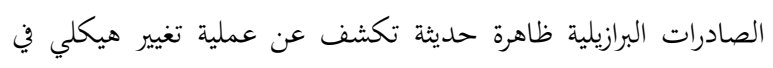

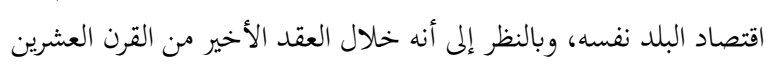

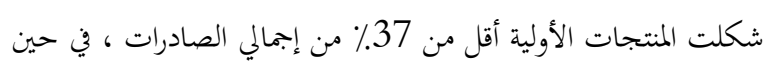

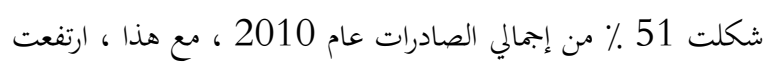

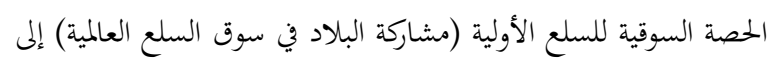

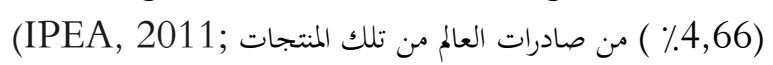

حتى مع ملاحظة السلطات الحكومية أن البلاد ستواجه أزمة من خلال تبني

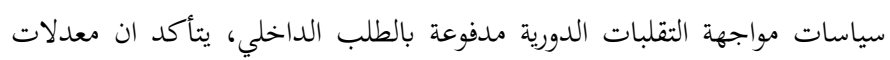

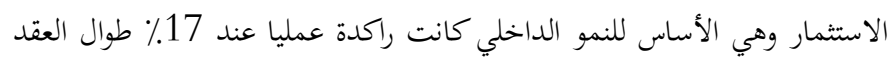

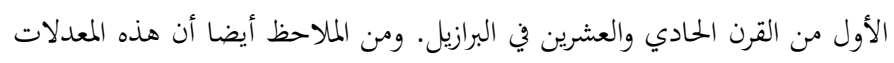

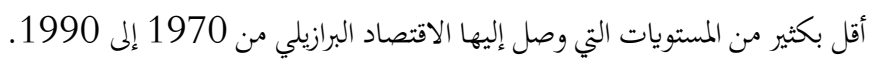

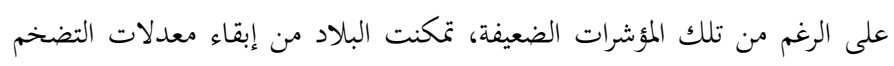

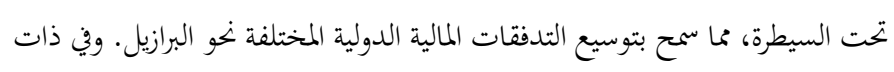

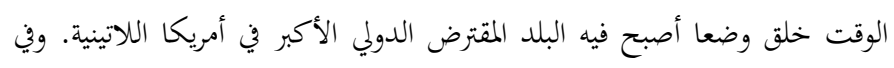

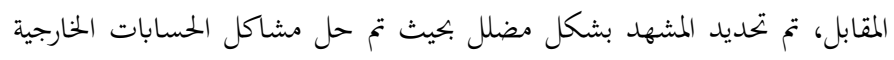

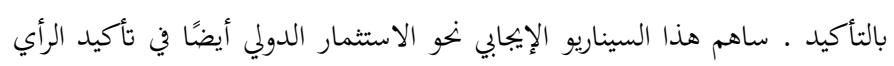

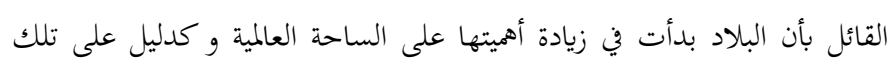

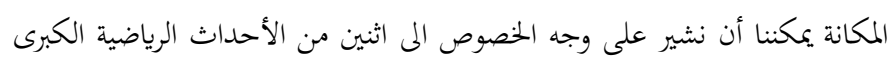

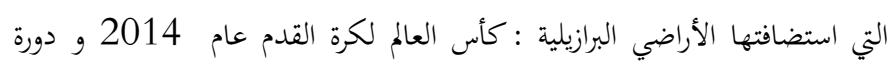

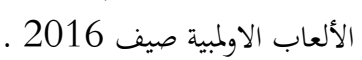
2.1 استراتيجية تعتمد على السوق الداخلي ساعد التوسع القوي للائتمان من بنوك الدولة، بالحفاظ على الدانلى النشاط

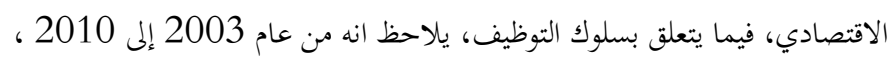

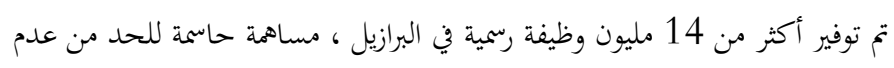

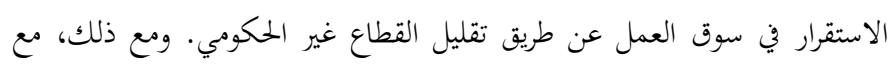

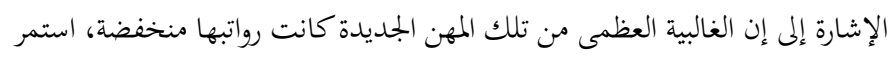

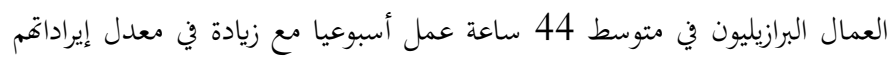

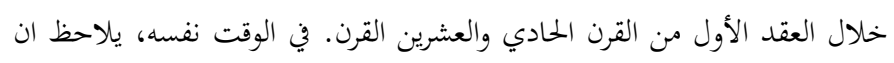

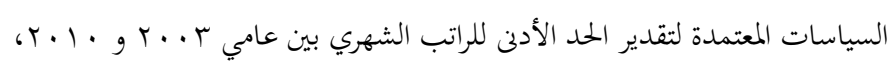

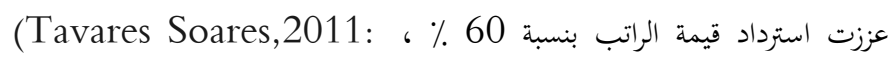
IPEA 2010a)

وعلى هذا النحو نما متوسط دخل الفرد البرازيلي الراكد لأكثر من عقدين بععدل

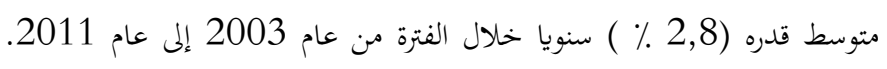

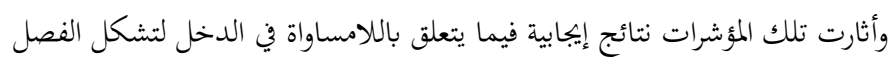
التاريخي بين متوسط الدخل في كلا الطرفين : اذ الخفض مؤشر عدم المساواة في الدخل

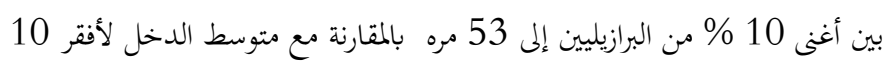

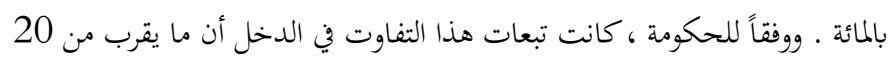

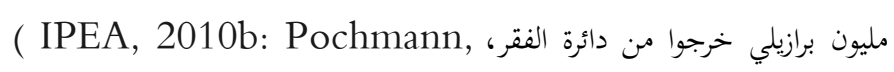

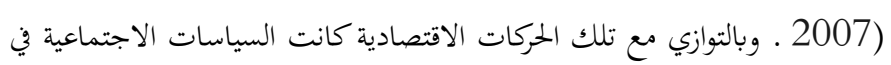

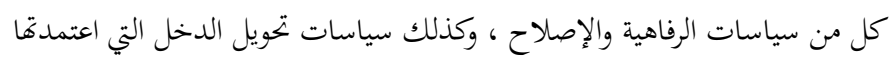

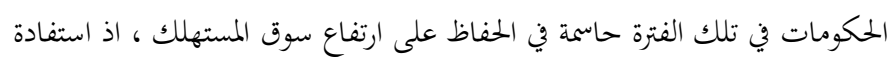

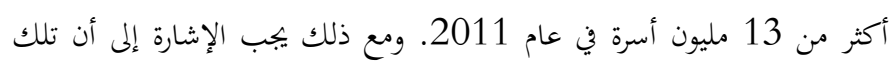

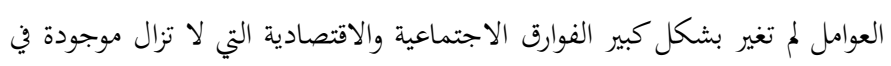

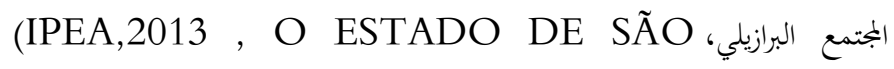
.PAULO, 20) 
مشروطة أكثر من أي وقت مضى بمركز الاقتصاد العلمي. وبينما يحدث

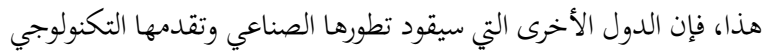

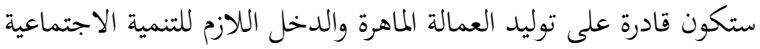

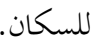
3- القطاع الزراعي: الاستمار العام و النتائج والأرقام والتناقضات الرئيسية

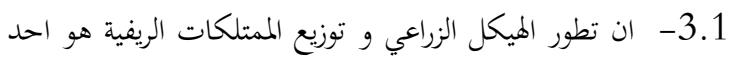

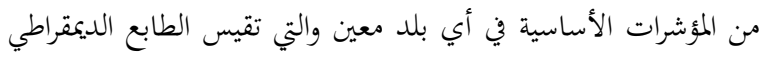

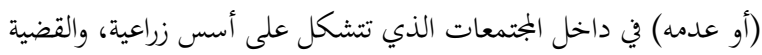

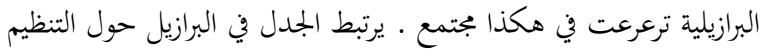

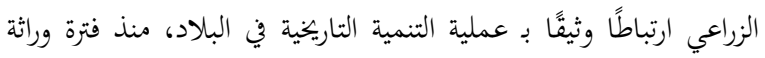

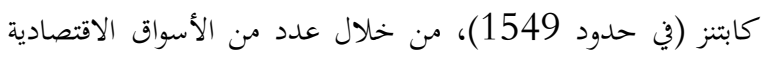

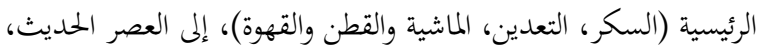

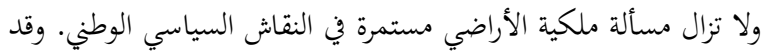
ساء هذا السيناريو بشكل ملحوظ في النصف الثاني من القرن العشرين

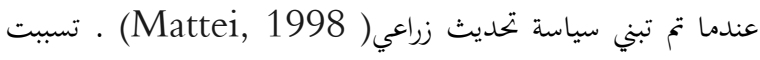
تلك العملية بتحولات عميقة في بحال الإنتاج الزراعي، كما أثارت سلسلة

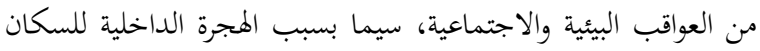

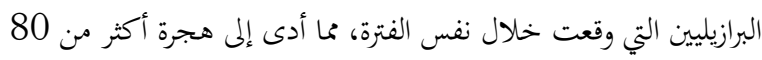

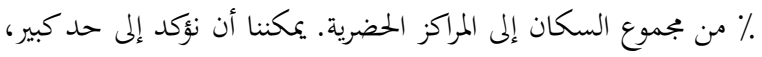
أن الصراع الاجتماعي الذي تم إدخاله في المناطق الريفية من البلاد يرتبط

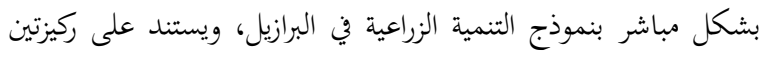

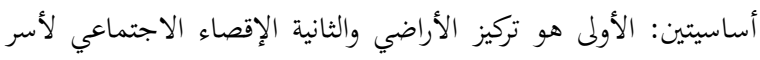

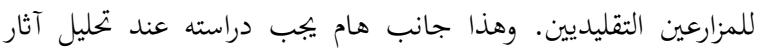

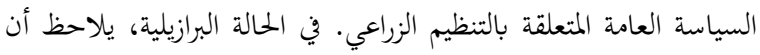

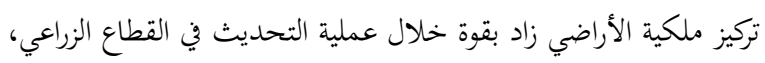
مع ذلك استمر التفاوت الشديد في الماضي والحاضر. التصنيف 1: تطور تركيز الأراضي في البرازيل (مؤشر جيني)

\begin{tabular}{|r|r|}
\hline YEARS & GINI INDEX \\
\hline 1975 & 0.800 \\
\hline 1985 & 0.820 \\
\hline 1995 & 0.856 \\
\hline 2006 & 0.872 \\
\hline
\end{tabular}

Source: Brazilian Institute of Geography and Statistics (IBGE, 2009).

وفقا للبيانات الإحصائية الرسمية، نما مؤشر جيني على مدى العقود الأربعة

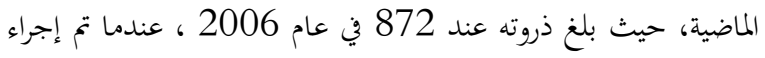

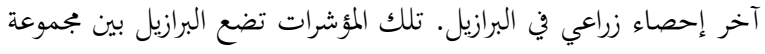

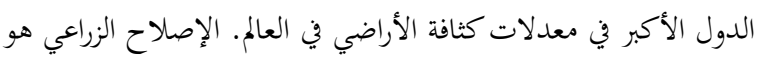

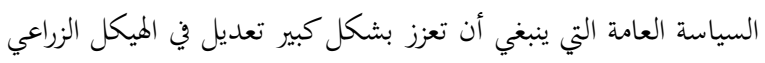

. Mattei, 2012; Governo Federal, 2010) الاقتصادية الحكومية فاية عام 2011 بتحقيق رقم قياسي آخر، حيث تم الإعلان

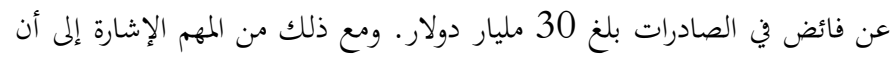

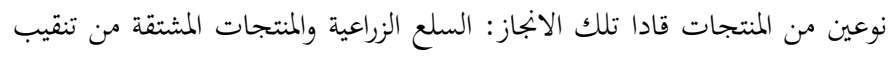

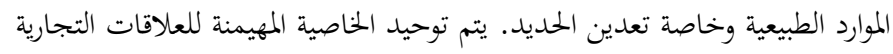
داخل الدولة على أساس تصدير المنتجات ذات المستويات المنخفضة من التكنولوجيا .

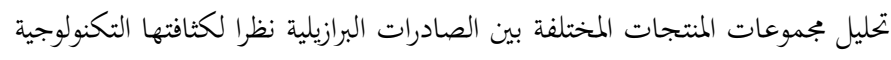

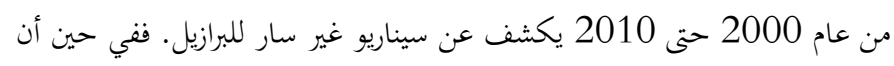
المنتجات ذات الكثافة التكنولوجية العالية انخفضت من 18 ٪ في عام 2000 إلى 200 إلى

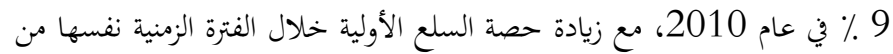

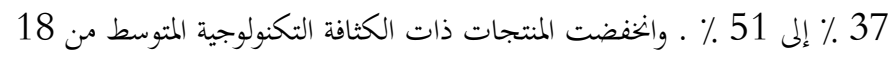

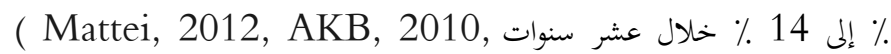
FUNDAP, 2011, Serrano \& Summa, 2011). الأرقام زيادة مشاركة الزراعة والتعدين على جدول منتجات الأعمال البرازيلي. حتى

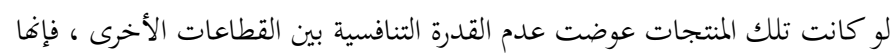

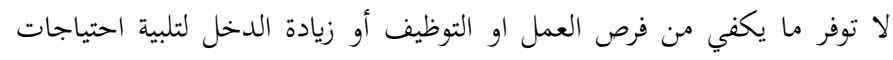

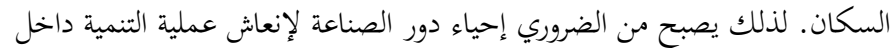

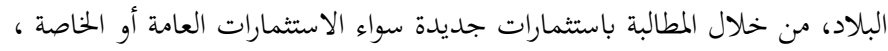
في المناطق التي تعد علمية أو تكنولوجية.

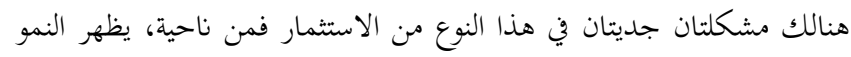

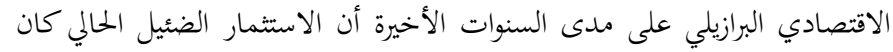

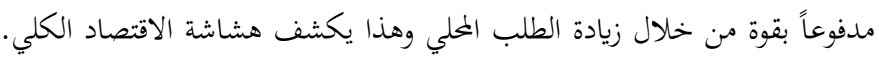

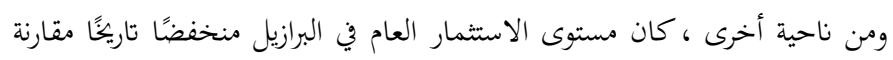

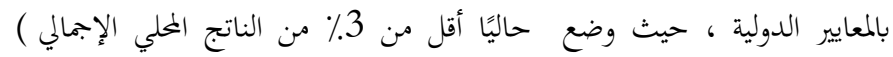
Governo Federal, 2010; Mattei, 2012; AKB, 2010). وفي العام الماضي زادت مشاركة المواد الخام الأساسية في الموازين التجارية البرازيلية،اذ

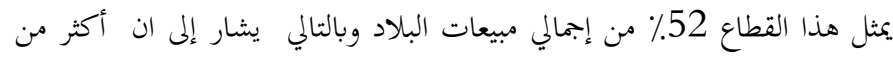

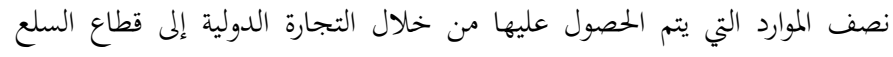

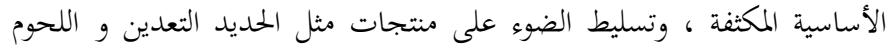

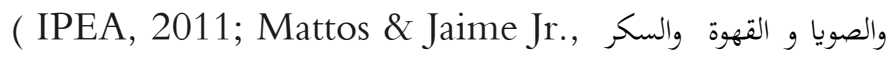

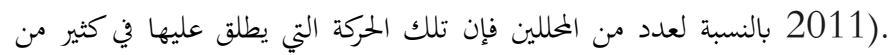

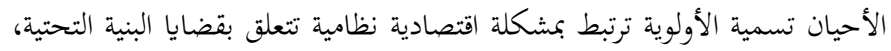

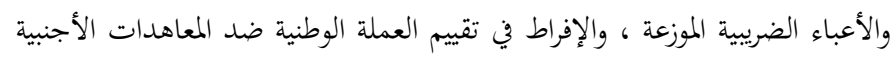

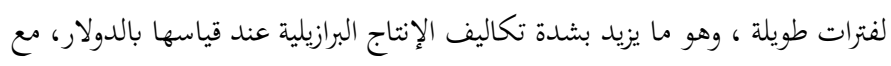
مراعاة ثبات العوامل الأخرى ـ تعد المبالغة في تقدير قيمة العملة العامل الأكثر أهمية،

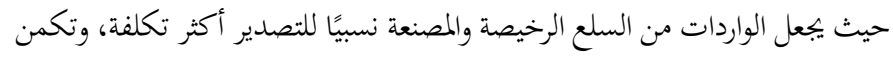

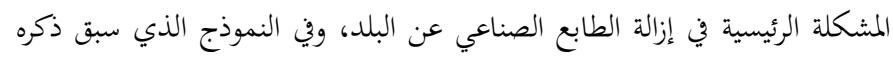

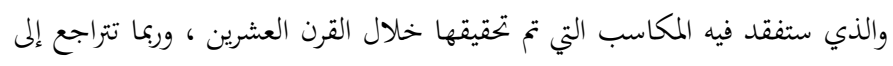
اقتصاد يعتمد على التصدير الأولي في القرن الحلادي والعشرين و تصبح ديناميكيتها 
من هذا المنطلق، يمكنا أن نؤكد أن جزء كبير من الأزمة الحضرية التي تفاقمت خلال العقدين الماضيين في كل منطقة من مناطق البرازيل تقرييا من خلال استيلاء غير منظم لأراضيها مع الضغط المستمر على الخدمات

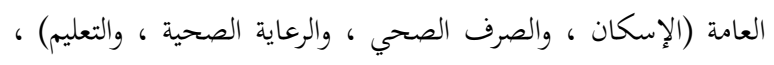

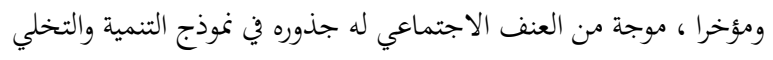

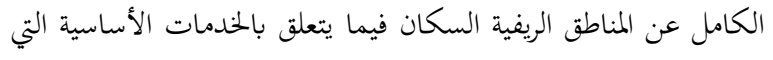

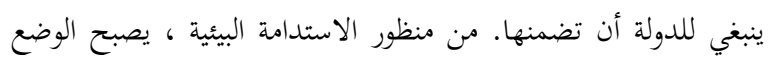

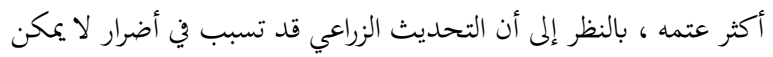

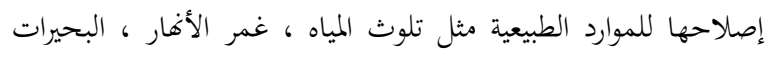

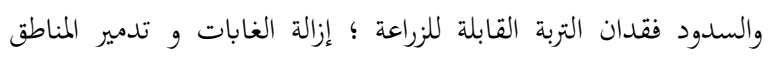

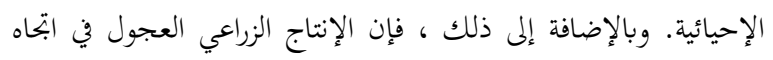

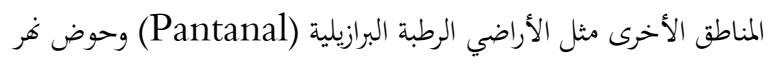
الأمازون وضع شكوكا أكبر على استدامة النموذج للأجيال القادمة.

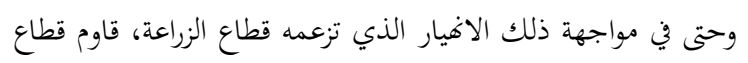

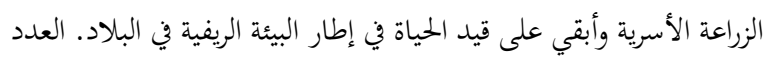

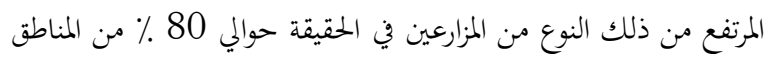

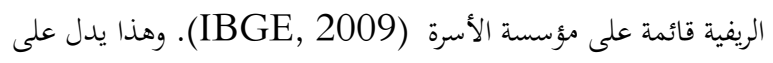
وجود نوع آخر من الزراعة لا يتبع نموذج التحديث ويمتلك منطقًا عمليًا

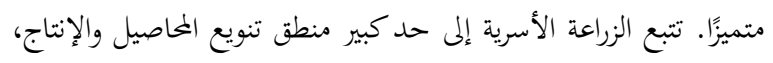

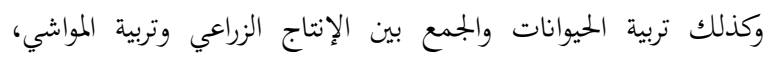

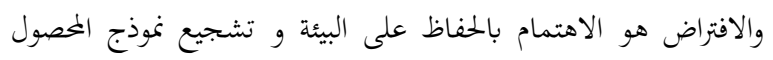

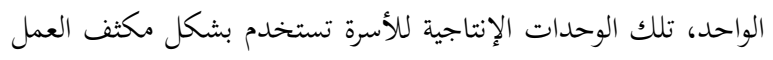

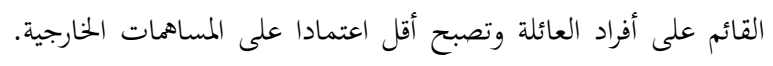

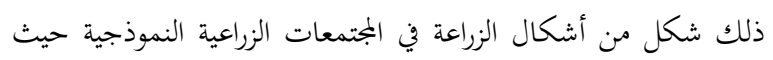

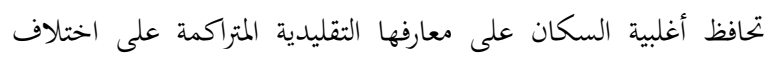

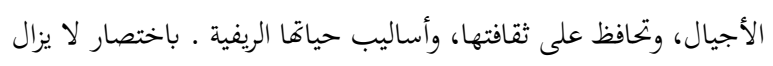

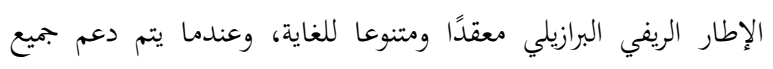

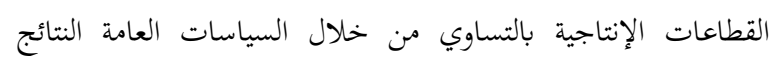

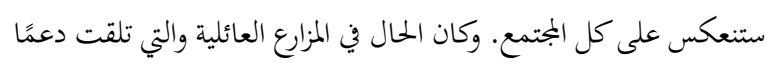

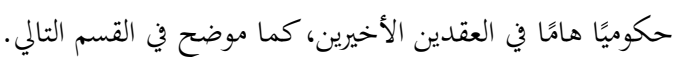
3.3- السياسات العامة لدعم المزارع الأسرية

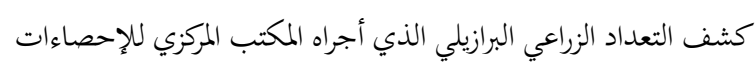

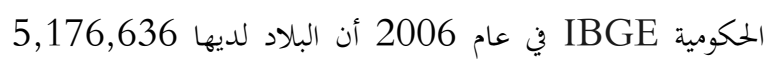
مؤسسة زراعية. صنفت من تلك

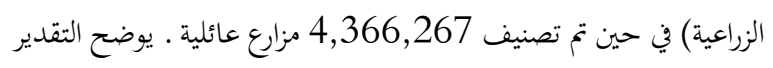

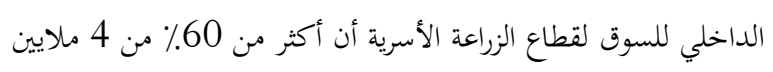

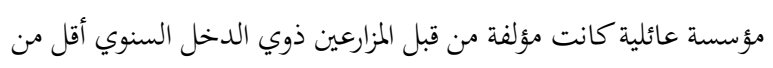

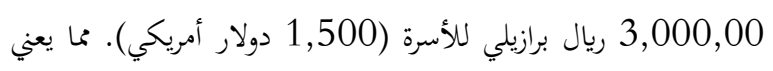

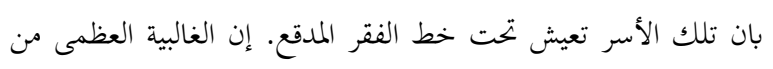

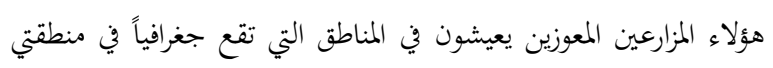

للبلاد، ويجب أن يتم ذلك بطريقة تجعل الوصول إلى الأرض ديمقراطيًا. ولسوء الحظ الحظ

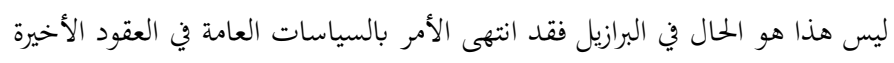

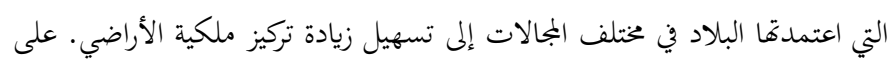

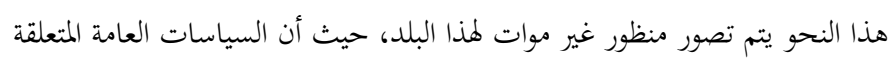

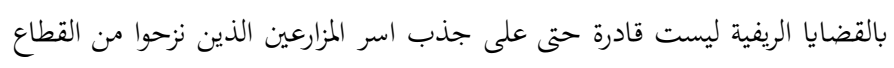

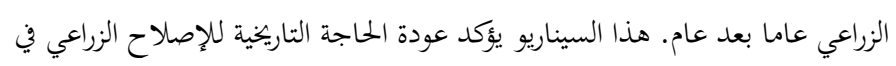
البرازيل كأجندة عامة.

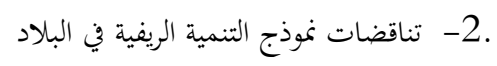

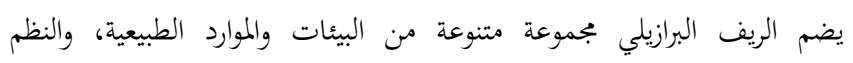

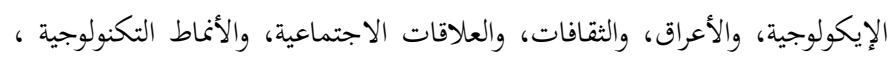

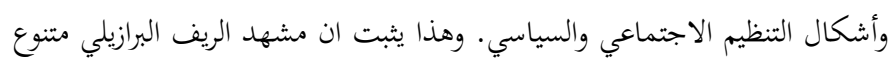

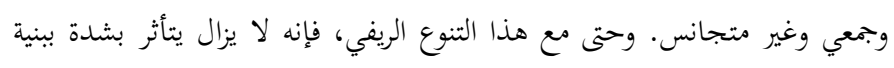

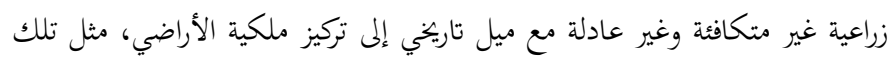

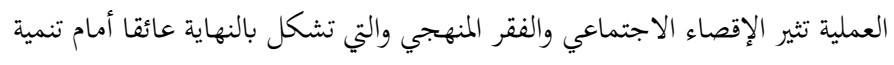

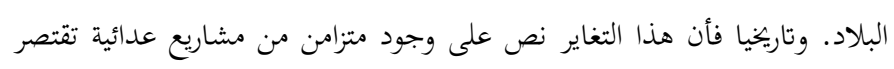
على نفس المساحة الجغرافية، فمن ناحية تستنسخ الزراعة التجارية (الأعمال الزراعية)

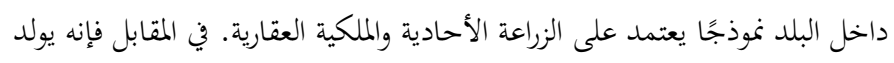

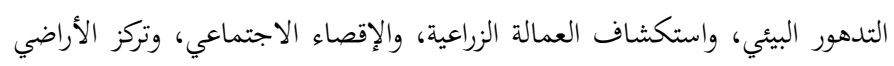

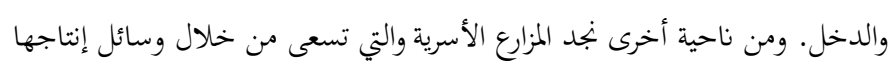

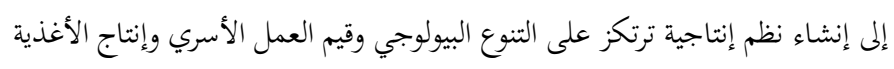

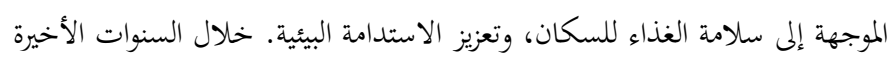

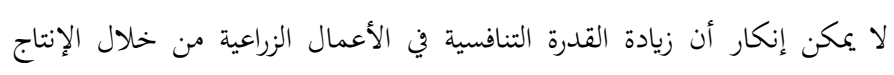

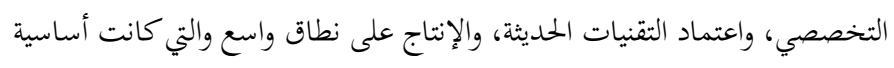

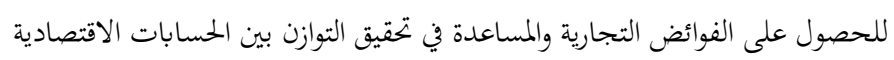

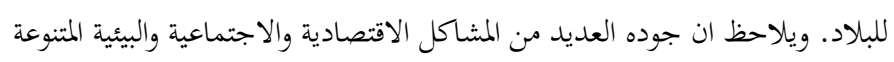

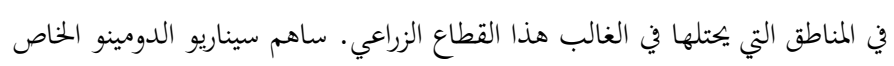

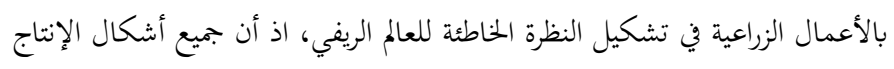

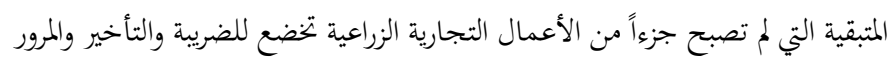

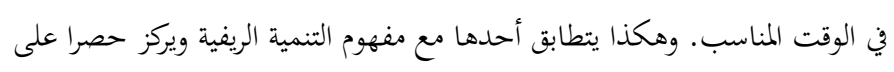

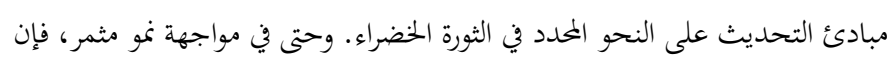

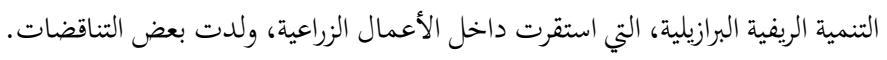

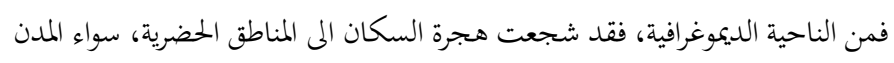
المتوسطة الحجم الداخلية او المراكز الحضرية الكبيرة وغو الأراضي الساحلية البرازيلية.

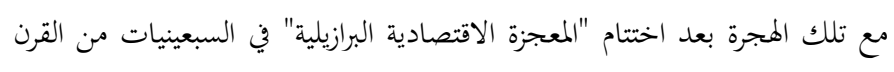

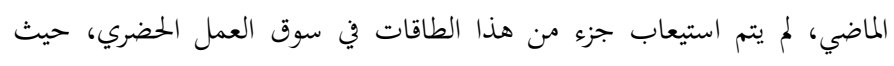

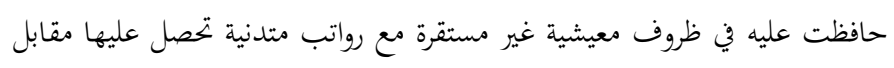

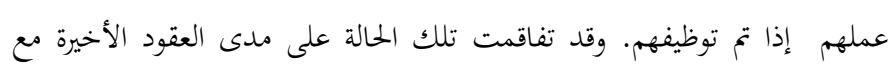

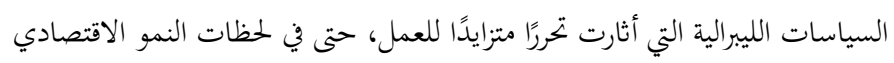
والزيادات في الاستثمارات الإنتاجية، لاسيما في قطاعات السلع الصناعية والزراعية. 
(Mattei, 2011) ، كانت نتائج تلك السياسة العامة فورية، ووفقاً

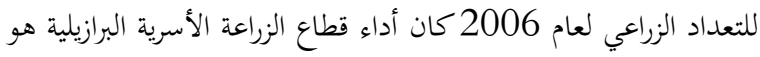

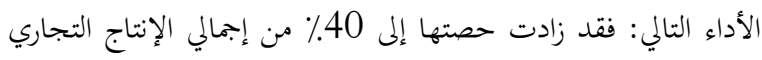

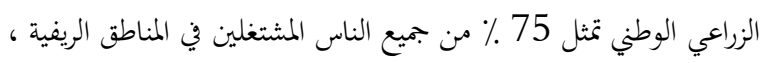

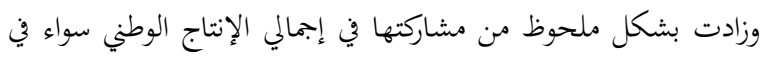

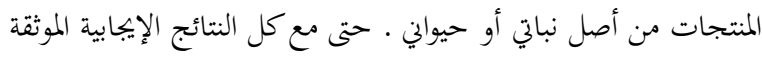

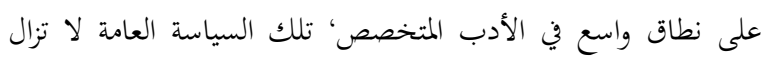

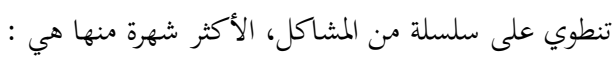

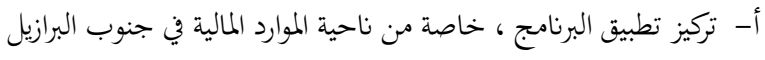
، في حين يتركز أكبر عدد من المزارعين الأسريين في المناطق الشمالية

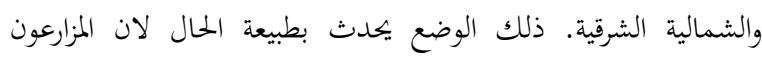
الأسريون في الجنوب أكثر تنظيما وأكثر مشاركةً في النظام المصريف.

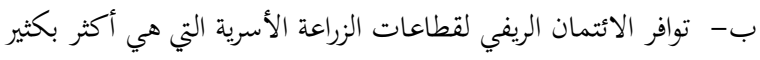

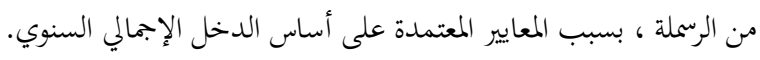

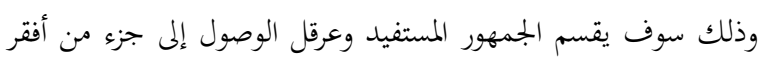

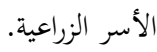

ج- استمرارية التحيز الإنتاجي والقطاعي ، وتحديد الآثار السياسية،

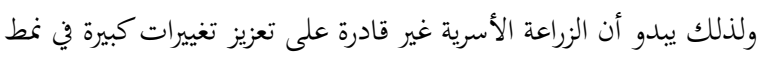

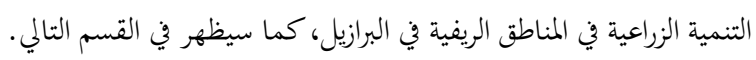
3.4 - الدعم الحكومي للأعمال الزراعية

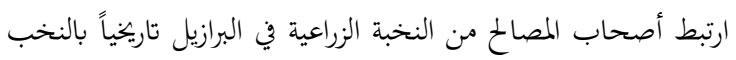

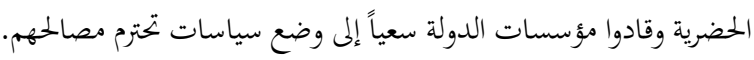

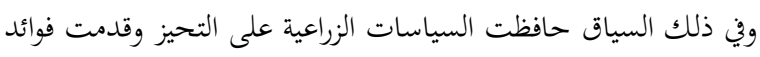

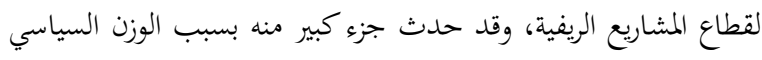

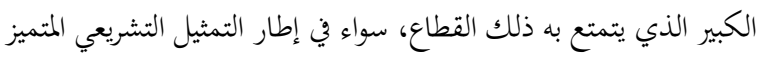

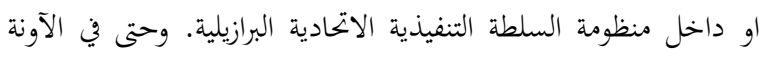

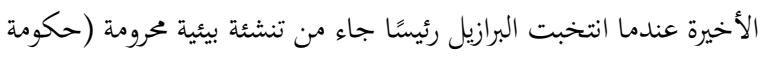

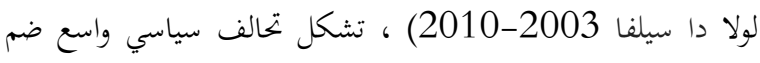

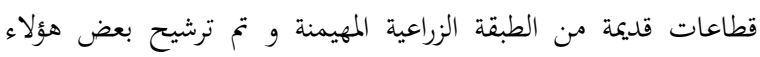

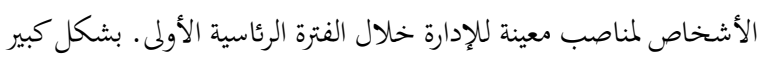

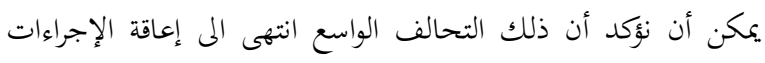

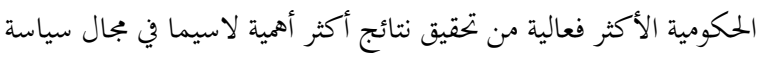

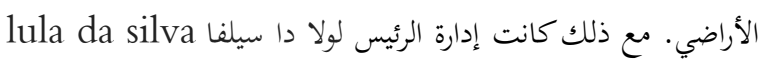

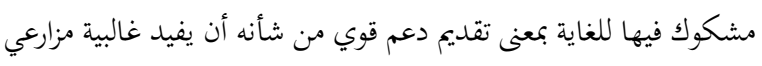

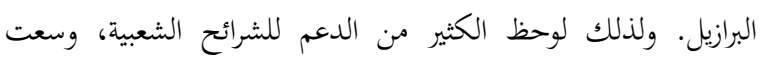

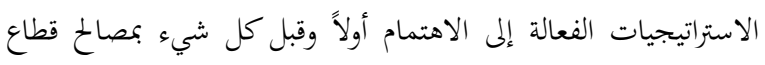

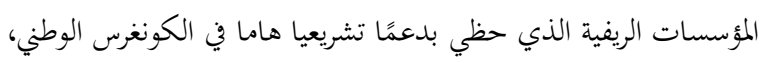
من خلال الحوافز والإعفاءات الضريبية.
شمال وشمال شرق البرازيل. وبعبارة أخرى تشكل المزارع العائلية 85٪ من إبمالي ملكية

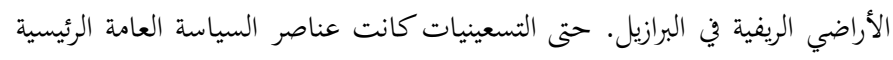

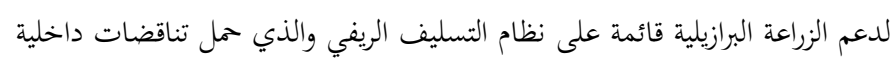

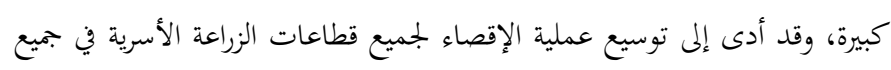

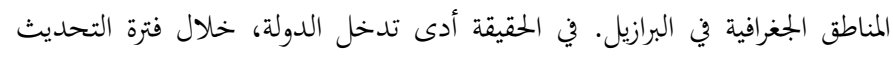

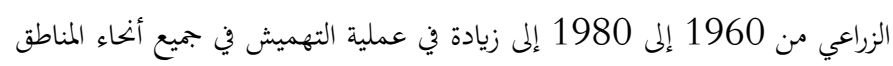

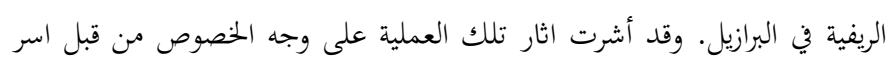

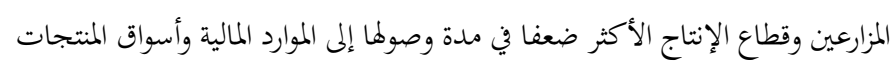
نفسها.

الزراعية

ولمواجهة المشكلة والتغلب عليه تم في عام 1996 إنشاء البرنامج الوطني لتدعيم الترائ الزراعة الأسرية (PRONAF) . كان هدفه هو تحفيز التوسع في الزراعة الأسرية

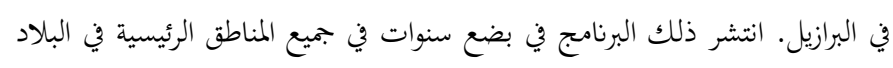

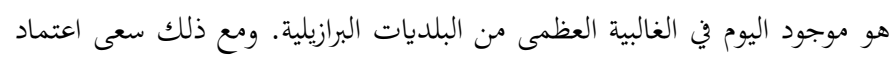

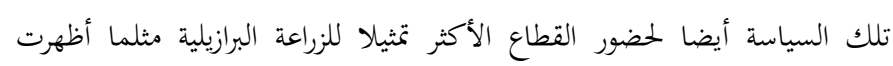

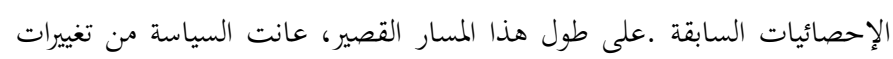

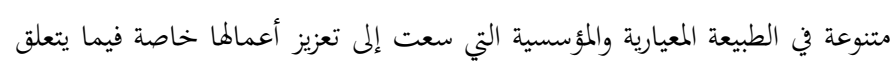

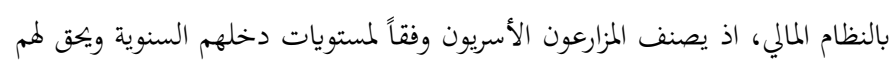
الحصول على التمويل بأسعار فائدة مناسبة مدعومة من الحكومة الفيدرالية البرازيلية.

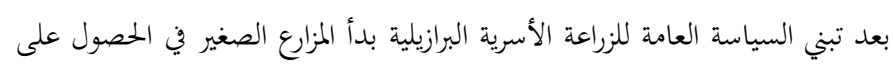

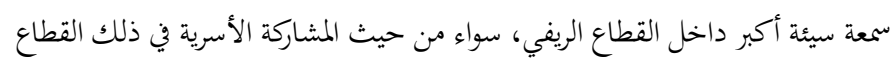

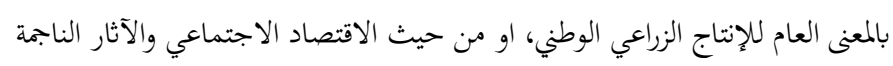

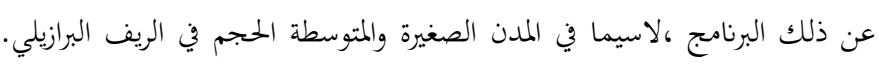

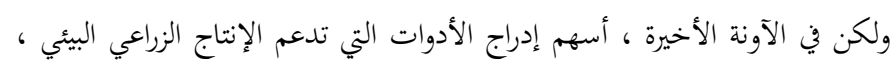

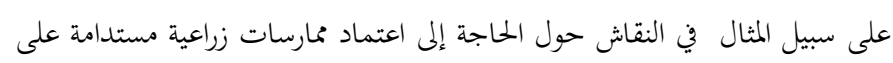
نطاق أوسع داخل البرازيل. مع مرور عشر سنوات أخرى من الخبرة في البناء، يلاحظ أن مشروع قد تم تقسيمه التحان.

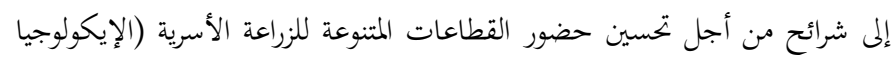

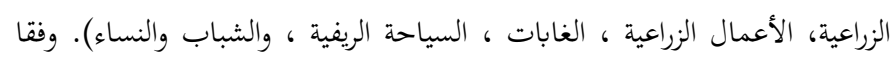

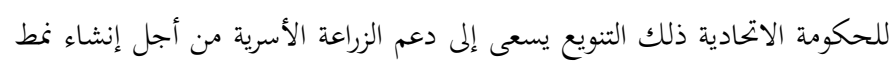

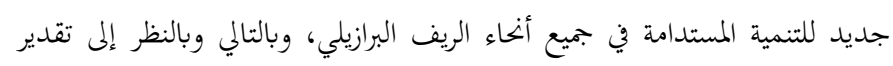

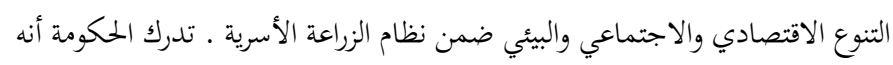

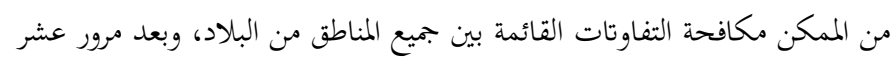

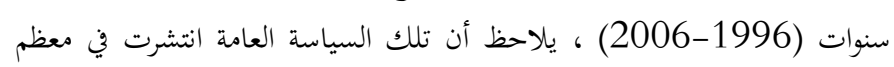

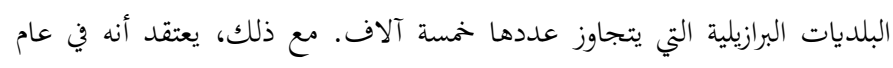

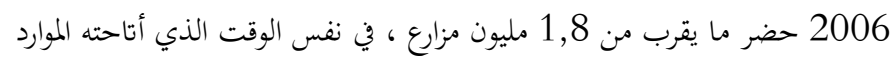

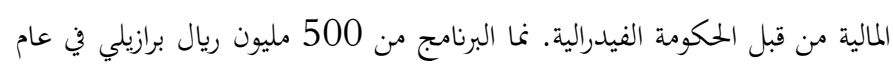
1996 إلى أكثر من 10 مليارات ريال برازيلي في عام 2006.

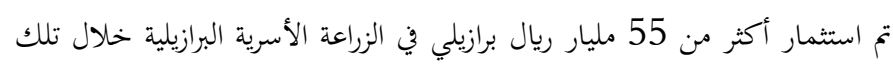

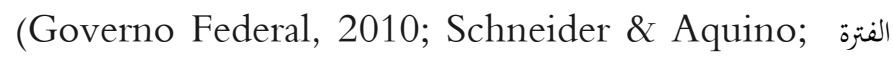


الصادرات من القطاع الريفي. كانت نتيجة تلك العملية عواقب سياسية مباشرة، لأن قطاع المؤسسات التي أنتجت مثل تلك الفوائض زادت من صلاحياتا السياسية التفاوضية داخل الكونغرس الوطني وكذلك الفرع التنفيذي الفيدرالي، مما ترك الإدارة الحكومية تحت رحمة مصالحها الخاصة. 4 أثارت المسألة البيئية استقطابًا حادًا بين القطاعين المشاركين في الإنتاج

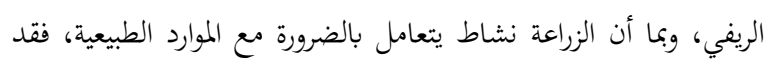
تدخلت كل من النظم الإنتاجية (المزارع العائلية والأعمال التجارية الزراعية)

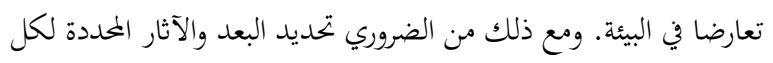

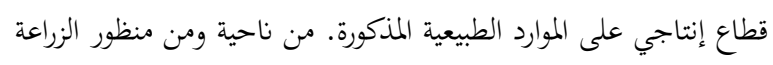

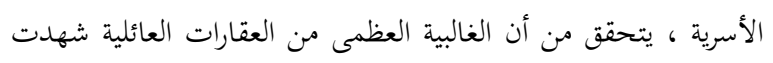

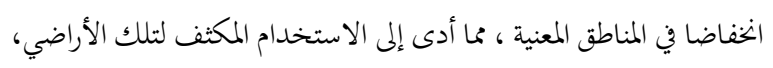
في تلك العملية ، يتم بشكل عام إزالة جميع المناطق المتاحة واستخدامها

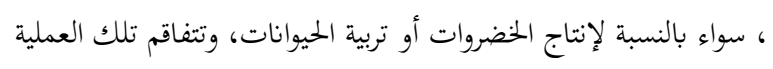
بسبب مشاكل الدخل المعتادة بالنسبة لتلك الشريحة من المزارعين الذين لديهم شروط محدودة للاستثمار في التقنيات الحديثة لحفظ الموارد الطبيعية . من ناحية أخرى ، مع التوسع الهائل في طبيعية النظم البيئية المتنوعة في

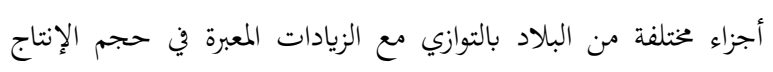

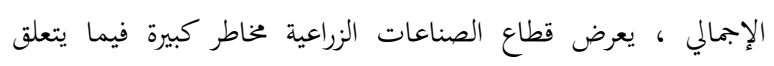
بالمستقبل البيئي للبرازيل. توجد أمثلة على ذلك في المناطق التي تسود فيها

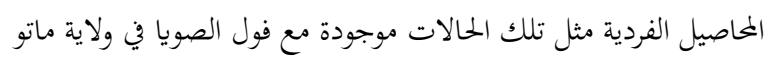

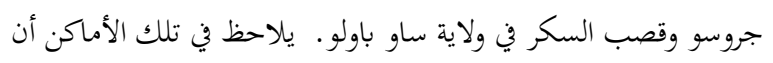

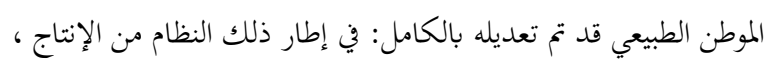

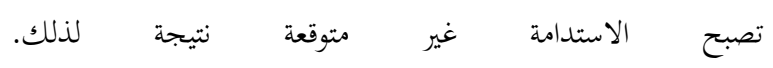
في ذلك العمل يتبين أن الزراعة الأسرية والأعمال الزراعية لا تختلف عن تصني

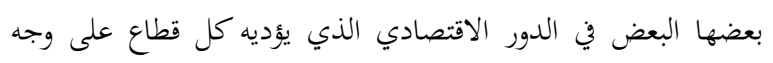
التحديد، ومع ذلك تحتاج التحليلات أيضًا إلى النظر في العناصر المهمة لتصني

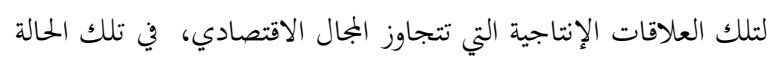

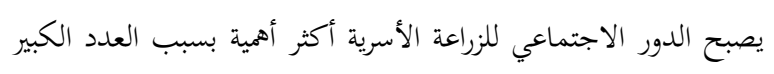

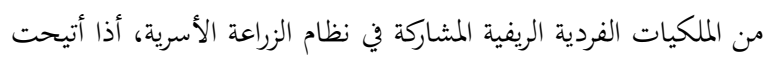

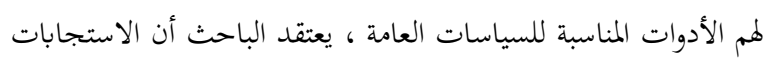

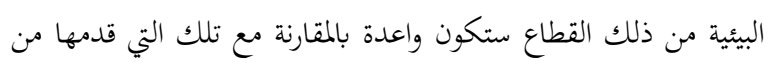
قبل نموذج إنتاج يخصص للاستكشاف الزراعي حصرا على أساس امتدادات واسعة من الأراضي معهم عدد قليل من الناس .

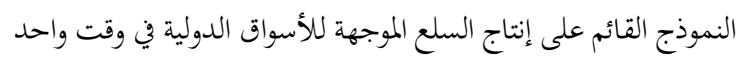
يثير عمليات متناقضة ومتضاربة ، فمن ناحية يحفز الأراضي الريفية الشاغرة

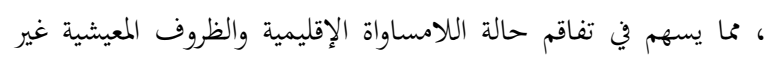
المستقرة لمعظم العائلات التي تعيش في المناطق التي عادة ما تكون ريفية، ومن ناحية أخرى فإن نظام الإنتاج القائم على المحاصيل الفردية والإنتاج

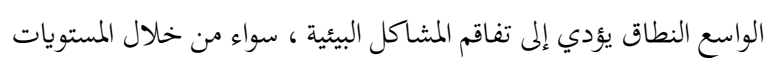

استفاد قطاع السلع الزراعية الإنتاجية الموجه نهو الأسواق الخارجية بشدة، ويف

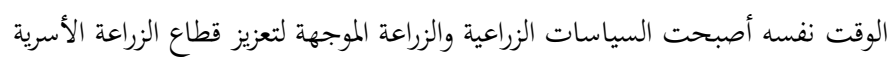
أكثر قمميشا في النظم الحكومية. ونتيجة لذلك كانت الإجراءات الحكومية الموجهة نحو الزراعة الأسرية ذات طبيعة تعويضية تقريبًا، دون أي قدرة حقيقية على تغيير المنطق

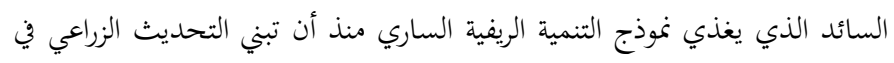
البرازيل. حافظ "المجلس القروي" المزعوم (Bancada Ruralista) وهو تعبير سياسي داخل الكونغرس الوطني يمثل تكتل مصالح قطاع المؤسسات الريفية في المشاركة السياسية التمثيلية خلال ولايتي إدارة لولا دا سيلفا المسؤولين عن الفرع التنفيذي في

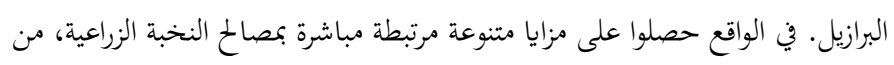
بين الإجراءات الرئيسية المعتمدة لصالح القطاع الريفي يمكن تسليط الضوء على الزيادة

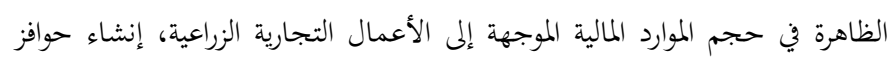
ضريبية لصادرات السلع الزراعية، رفع القيود عن المحاصيل المعدلة وراثيا (GMOs)

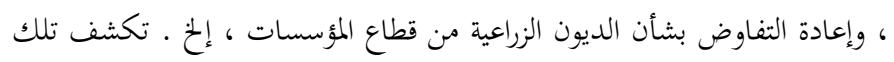

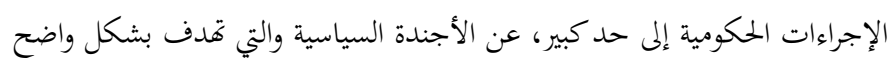

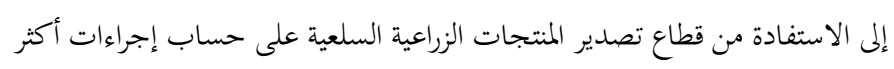

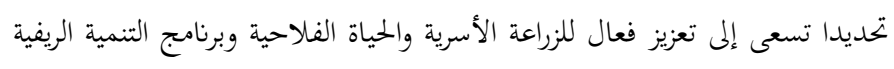
المستدامة ذاته الذي تقوم به الحكومة. كانت نتيجة تلك الإجراءات خلال تلك الملدة

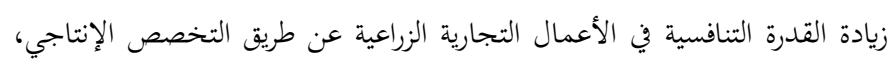

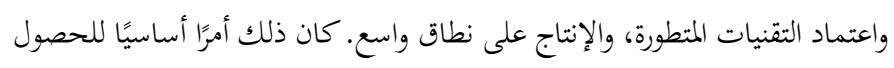
على أرصدة مواتية في حسابات التجارة الخارجية، مما ساعد على موازنة الحسابات الخارجية للبرازيل. ومع ذلك في المناطق التي تهيمن فيها الأعمال التجارية الزراعية، كما

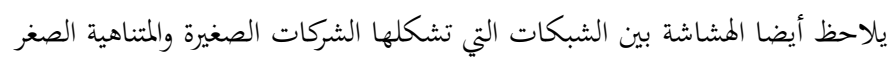

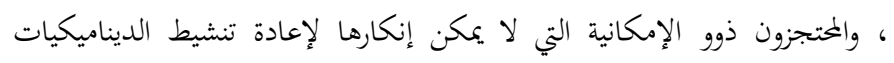
الاقتصادية المحلية.

لم يسهم نمو وتكثيف الزراعة كثيراً في تنويع سلع المجتمعات الريفية. وبالنظر إلى نمو أكثر من 70٪ في الإنتاج الإجمالي للحبوب خلال العقود الأخيرة كمؤشر وحيد

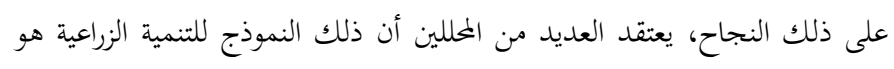

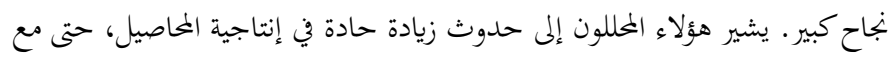
تكاليف تكثيف ممارسات المحاصيل الفردية وتوسيع الاعتماد على المدخلات الكيميائية

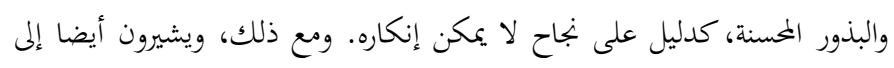

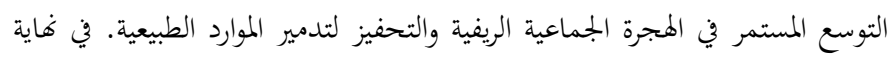
عام 2011 احتفلت السلطات الاقتصادية الحكومية بتسجيل رقم قياسي مع فوائض

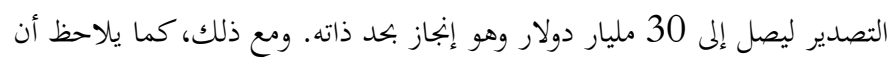

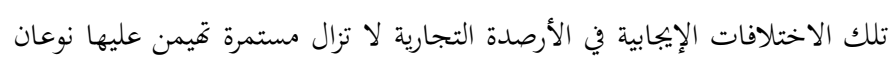

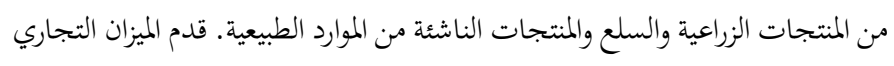
البرازيلي في نفس العام أحد أكبر الفوائض في تاريخه، معتمداً على المشاركة الحاسمة

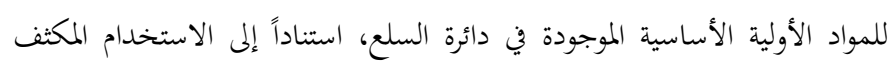

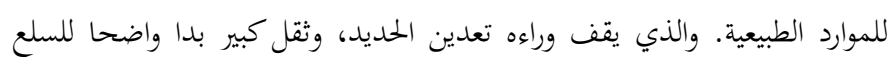

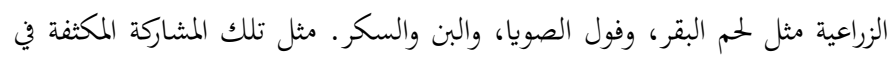

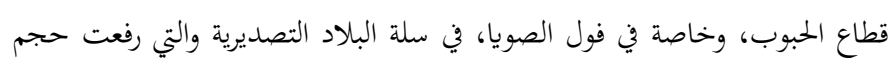


FUNDAP. Boletim de Economia, n.3, abril de 2011. [9]

GOVERNO FEDERAL. Ministério da Fazenda, Boletim Economia [10] Brasileira em Perspectivas. Ediçao Especial, 2010. STICA ZINSTITUTO BRASILEIRO DE GEOGRAFIA E ESTAT [11] fico de 2010. Rio de Janeiro: IBGE, 2011.J(IBGE). Censo Demogr STICA ZINSTITUTO BRASILEIRO DE GEOGRAFIA E ESTAT [12]

rio de 2006. Rio de Janeiro: IBGE, 2009.J(IBGE). Censo Agropecu INSTITUTO DE PESQUISA ECONoMICA APLICADA. Revista [13] Desafios do Desenvolvimento, ano 8, n.66, 2011.

INSTITUTO DE PESQUISA ECONoMICA APLICADA. Revista [14] Desafios do Desenvolvimento, ano 9, n.73, 2012.

INSTITUTO DE PESQUISA ECONoMICA APLICADA. Revista [15] Desafios do Desenvolvimento, ano 10, n. 77, 2013

INSTITUTO DE PESQUISA ECONoMICA APLICADA. Pobreza, [16] desigualdade e politicas publicas. In: Comunicados da Presidência, n. 38, Janeiro de 2010. o, pMICA APLICADA. DimensiNSTITUTO DE PESQUISA ECON [17] o da pobreza por regiues e estados no Brasil. In: po e projeç̧evoluç Comunicados da Presidência, n. 58, Julho de 2010

LAYARGUES, P. P. Do Ecodesenvolvimento ao Desenvolvimento [18] Sustentivel: Evoluçao de um conceito. 2007.

MATTEI, L. A evoluçao do emprego agrucola no Brasil. Sao Paulo: [19] o ABETMercado de Trabalho, 1998.pColę̧

lia: يMATTEI, L. Impactos do Pronaf: anilise de indicadores. Bras [20] MDA/NEAD, 2005.

MATTEI, L. Mudanças nas regras de financiamento do PRONAF. Rio [21] de Janeiro: OPPA/CPDA/UFRRJ, n. 17, março de 2008.

MATTEI, L. Evoluçao do crédito do PRONAF para as categorias de [22] agricultores A e A/C entre 2000 e 2010 In: Anais do 49 Congresso da SOBER. , 2011.

MATTEI, L. Brazilian development at beginning of the 21st Century: [23] economic growth, income distribution, and environmental destruction. In "Inside a Champion: an analysis of the Brazilian development model". Berlin, Heinrich Boll Stiftung, 2012.

MATTOS, F.A.M. \& JAIME JR, F.G. Ganhos sociais, inflexues na [24] tica econômica e restriçao externa. In: Revista Economia \& spol

Tecnologia, n.7, ediçao especial, 2011.

tica. Rio de JMAY, P. H. Economia do Meio Ambiente. Teoria e pr [25] Janeiro: Elsevier, 2010

MEADOWS, D. Et Al. The limits of Growth. A report for the club of [26] Rome's project on the predicament of Mankind. Londres:Potomac, 1974

PRET, R. C. Economia verde e commoditizaçao da vida. In: Revista [27] NECAT, ano 1, n.2, julho-dezembro de 2012

POCHMANN, M. Brasil, o pais dos desiguais. In: Lê Monde [28] Diplomatique, ano $1, \mathrm{n} .3$, outubro de 2007.

tica da sustentabilidade? sROMEIRO, A R. Economia ou economia pol [29] In: MAY, Peter. Economia do Meio Ambiente. Teoria e pratica. 2 ed. Rio de Janeiro: Elsevier, 2010 ROMEIRO, A R. Economia ou economia politica da sustentabilidade? [30]

Texto para Discussao.IE/UNICAMP, Campinas, n. 102, set. 2001. vel. Rio de JSACHS, I. Caminhos para o Desenvolvimento Sustent [31] Janeir: Editora Garamond, 2009.

SCHNEIDER, S. \& AQUINO, J. 12 anos de polatica de crédito do [32] PRONAF no Brasil (1996-2008): uma reflexao critica. Campo Grande Congresso Brasileiro da SOBER, 2010.؛(MS): 48 ticas SSCHNEIDER, S. A importância da pluriatividade para as pol [33] lia, ano XVI, يcola. Brasstica Agr spublicas no Brasil. Revista de Pol n. 3, p. 14-33, jul./ago./set. 2007.

SEQUINEL, M. C. M. Cupula mundial sobre desenvolvimento [34] vel. ANaLISE ssustentavel - Joanesburgo: entre o sonho e o poss CONJUNTURAL, v.24, n.11-12, p.12, nov./dez. 2002.

SERRANO, F. \& SUMMA, R. Politica Macroeconômica, crescimento e [35] distribuiçao de renda na economia brasileira dos anos 2000. In Observaturio da Economia Global, n.6, março de 2011.

TAVARES SOARES, M.A. Salario Minimo e o paradoxo da reduçuo [36] da desigualdade no Brasil. In: Revista Oikos, v.10, n.2,
المرتفعة لإزالة الغابات وتحويل المناظر الطبيعية إلى بيئات موحدة موجهة نحو الإنتاج الزراعي على نطاق واسع أو من خلال عملية التدهور المتراكمة للمحميات الطبيعية ،وهي الوسيلة الوحيدة لبقاء السكان التقليديين ، مثل قبائل السكان الأصليين وأحفاد العبيد البرازيليين الهاربين الكويلومبولا (quilombola) . يسمح لنا ذلك السيناريو بأن نؤكد أن المحيط الريفي البرازيلي لا يزال يتسم بالهيكلية من خلال تركيز الثروة، والهيمنة السياسية والاقتصادية على الأوليغاركية التقليدية، وباعتماد الشركات عبر الوطنية والأسواق الدولية، وبالتالي على المصالح الاجتماعية والإقليمية العميقة. عدم المساواة التي تولد الإقصاء الاجتماعي والفقر الممنهج حتى لو اكتسبت السمات

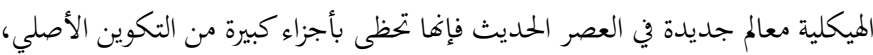
وبالتالي فإن عمليات التدويل والاحتكار في عمليات الإنتاج الزراعي، والتغيرات في بئي المعايير التكنولوجية، وتقييم المنتجات المشتقة من الزراعة والتوسع الكبير للحدود الزراعية يُكشف عن استمرارية الاعتماد على النموذج أشكال المهيمن من الإنتاج

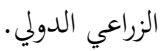

يستنتج البحث أن تلك التحليلات يجب أن تدرك حقيقة أن الانتقال من بجتمع

زراعي إلى بجتمع صناعي، عملية حدثت في البرازيل في القرن العشرين، والتي عززت

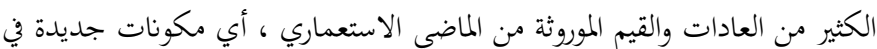
عملية التنمية الوطنية دون تغيير مسار التاريخ. ومع ذلك فإن المسار الذي رسمه المجتمع البرازيلي منذ تأسيسه يتسم بالتناقض بين والوفرة لعدد قليل من السكان والمجاعة للغالبية العظمى. من المهم تسجيل أن التناقض قد تعزز خلال فترة ما بعد الحرب وقاد البرازيل إلى الرقم بين أكثر البلدان تناقضًا في العالم، فعلى الرغم ان عام 2011 أظهر البرازيل كسادس أكبر اقتصاد في العالم، ولكنها في الوقت ذاته استمرت في كوها دولة ذات التفاوتات الأكبر في العالم وأكبر المعدلات من الإقصاء الاجتماعي. إن موضوعا الفقر والبؤس أصبحا في الآونة الأخيرة من الأولويات في أجندة العمل السياسي الوطني واللذان يعكسان بالضبط ذلك الوضع المتناقض تاريخيا. وهكذا، فإن الاستنتاج الرئيسي هو أن تلك هي المشاكل الاجتماعية والسياسية هي بالضبط التي لا تزال تعوق المجتمع البرازيل نحو وضع أكثر عدلا ومساواة في البيئتان الريفية والحضرية.

ASSOCIAO KEYNESIANA BRASILEIRA (AKB). Dossiê Crise II. Porto Alegre (RS): AKB, 2010

BITTENCOURT, G. Abrindo a caixa preta: o financiamento da agricultura familiar no Brasil. Campinas, 2003. 222f. Dissertaçao (Mestrado em Desenvolvimento Econômico, Espaço e Meio Ambiente) - Instituto de Economia, Universidade Estadual de Campinas, 2003.

A.; EYRE, N.; HOWAR, N. Carbon Markets, an International Business əBROH Guide. Earthscan. 2009.

COCHRAN , I.T. \& LEGUET , B. Carbon Investment Funds: The Influx of Private Capital. Note d'étude de la Mission Climat de la Caisse des Dêpots. Research Report n 12, November 2007

RICA LATINA E CARIBE (CEPAL). ¿O ECONOMICA PARA AMICOMISS

Cambio estructural para la igualdad. Santiago de Chile: CEPAL, Julio de 2012 DALY, H. Crescimento Sustentvel Nao, obrigado. In: The case against the global economy. Sao Francisco; Sierra Club Books, 1996.

DAILY, H. \& FARLEY, J. Economia Ecolugica: principios e aplicaçues. Lisboa: Instituto Piaget, 2004

O PAULO. Brasil é o terceiro pior do mundo em desigualdade. IO ESTADO DE S Caderno de Economia, Julho de 2010 